Fatemeh Norouzi* and Gaston M. N'guérékata

\title{
Existence results to a $\psi$ - Hilfer neutral fractional evolution equation with infinite delay
}

https://doi.org/10.1515/msds-2020-0128

Received December 8, 2020; accepted March 30, 2021

\begin{abstract}
In this paper, we prove the existence and uniqueness of a mild solution to the system of $\psi$ - Hilfer neutral fractional evolution equations with infinite delay ${ }^{H} \mathbb{D}_{0^{+}}^{\alpha, \beta ; \psi}\left[x(t)-h\left(t, x_{t}\right)\right]=A x(t)+f\left(t, x(t), x_{t}\right), \quad t \in$ $[0, b], \quad b>0$ and $x(t)=\phi(t), \quad t \in(-\infty, 0]$. We first obtain the Volterra integral equivalent equation and propose the mild solution of the system. Then, we prove the existence and uniqueness of solution by using the Banach contraction mapping principle and the Leray-Schauder alternative theorem.
\end{abstract}

Keywords: $\psi$-Hilfer fractional derivative, infinite delays, mild solution, Banach contraction principle, LeraySchauder alternative

MSC: 34A08, 34A12, 34G99, 34K99

\section{Introduction}

In this paper, we consider the following $\psi$-Hilfer neutral fractional differential equations with infinite delays:

$$
\left\{\begin{aligned}
{ }^{H} \mathbb{D}_{0^{+}}^{\alpha, \beta ; \psi}\left[x(t)-h\left(t, x_{t}\right)\right] & =A x(t)+f\left(t, x(t), x_{t}\right), & & t \in[0, b], \quad b>0 \\
x(t) & =\phi(t), & & t \in(-\infty, 0]
\end{aligned}\right.
$$

where ${ }^{H} \mathbb{D}_{0^{+}}^{\alpha, \beta ; \psi}($.) is the $\psi$-Hilfer fractional derivative of order $0<\alpha \leq 1$, with respect to function $\psi \in$ $\mathcal{L}([0, b], \mathbb{X})$ and type $0 \leq \beta \leq 1$. Also, $x(t) \in \mathbb{X}$ is the state vector, $h(t)$ is a continuous function where $h:[0, b] \times \mathcal{P} \longrightarrow \mathbb{X}, t \in[0, b]$ and $\phi(t) \in \mathcal{P}$, where $\mathcal{P}$ is the admissible phase space. $f:[0, b] \times \mathbb{X} \times \mathcal{P} \longrightarrow \mathbb{X}$ and $f \in C^{1}$ is a nonlinear function and $x_{t}$ stands for the history of the state function up to the time $t$, i.e. $x_{t}(\theta)=x(t+\theta) \quad \forall \theta \in(-\infty, 0]$.

Let $A$ be an infinitesimal generator of an analytic semigroup $\{T(t)\}_{t \geq 0}$ of uniformly bounded linear operators defined on infinite Banach space $\mathbb{X}$. We denote $\mathcal{C}=\mathcal{C}_{b}((-\infty, b], \mathbb{X})$ the Banach space of all continuous and bounded functions from $(-\infty, b]$ into $\mathbb{X}$ endowed with the topology of uniform convergence.

$$
\|u\|_{e}=\sup _{t \in(-\infty, b]}\|u(t)\|
$$

and let $\left(\mathcal{B}(\mathbb{X}),\|\cdot\|_{\mathcal{B}(\mathbb{X})}\right)$ be the Banach space of all linear and bounded operators from $\mathbb{X}$ to $\mathbb{X}$.

Remark 1. Since $\{T(t)\}_{t \geq 0}$ is an analytic semigroup on $\mathbb{X}$, there exists $K \geq 1$ such that $\|T(t)\| \leq K$. (For more information on semigroups please see [4]).

\footnotetext{
^Corresponding Author: Fatemeh Norouzi: Department of Mathematics, NEERLab Laboratory, Morgan State University, Baltimore, MD 21251, USA, E-mail: Fatemeh.norouzi@morgan.edu

Gaston M. N'guérékata: University Distinguished Professor, Department of Mathematics, NEERLab Laboratory, Morgan State University, Baltimore, MD 21251, USA, E-mail: Gaston.N’Guerekata@morgan.edu
} 
In recent years, fractional calculus has been used and known as an excellent instrument for the description of hereditary properties of various processes and applications in many fields such as physics, biology, finance, etc. [6, 20, 24]. In the recent decades, many scientific researchers have become interested to study and investigate the existence and uniqueness of solution to the system of fractional differential equations. As one of the special and important problems in fractional calculus, there were some studies on the neutral type differential equations. Some of the authors proved the existence and uniqueness of the solution to semilinear neutral type fractional differential equations with infinite delays by using the Banach Contraction mapping theorem such as $[19,26]$. In $[2,14]$, researchers investigated the existence of solution by applying the Kuratowski's measure of noncompactness. Also, in [18], the authors proved the existence and uniqueness of solution using Banach fixed point theorem and the Leray-Schauder Alternative Theorem.

In finite dimensional case, the existence and uniqueness of solutions to problems of type (1) are widely studied in [26] by means of Schauder's fixed point theorem. Moreover, Zhou and Jiao [27] investigated the existence of nonlocal Cauchy problem by applying the Krasnoselskii's fixed point theorem and Banach fixed point theorem.

Recently, general fractional operators, especially such as the $\psi$-Hilfer fractional operator and the RiemannLiouville fractional operator with respect to another function have been proposed. Then in 2018, Vanterler and Capelas de Oliveira [23] presented a fractional differential operator of a function with respect to another function, the so-called $\psi$-Hilfer derivative. Obviously, the class of fractional derivatives derived from the $\psi$-Hilfer operator is in fact larger, making the fractional operator a generalization of the fractional operators. There are some authors who have worked on the existence and uniqueness of $\psi$-Hilfer and $\psi$-Caputo fractional differential equations as in [22]. In addition, Liu, et al. [15] presented some results on the existence, uniqueness, and Ulam-Hyers-Mittag-Leffer stability of solutions to a class of $\psi$-Hilfer fractional-order delay differential equations. They used the Picard operator method and a generalized Gronwall inequality involved in a Riemann-Liouville fractional integral. Also, Abdo, et al. [1] considered the fractional integro-differential equation with nonlocal condition involving a general form of Hilfer fractional derivative. They proved that Cauchy-type problem is equivalent to a Volterra fractional integral equation and used the Banach fixed point theorem and Krasnoselskii's fixed point theorem to obtain existence and uniqueness of solutions. Moreover, Luo, etc. [16] proved the existence and uniqueness of solution by Banach contraction mapping principle and investigated the Ulam-Hyers stability of solutions.

Motivated by all the above mentioned, we are interested to consider the system (1) of $\psi$ - Hilfer neutral fractional evolution equations with infinite delay which have not been used to be investigated. In our work, we aim to investigate and prove the existence and uniqueness of solution by means of Banach contraction mapping principle and the Leray-Schauder alternative theorem. In this case, first we have obtain the Volterra integral equivalent equation and propose the mild solution of system (1) in finite dimensional space.

The rest of our paper is organized as follows: In section 2 we present some preliminary results that are useful in this paper to prove the results. In Section 3, we prove the existence and uniqueness of solution by using Banach contraction mapping principle which states that there exists a unique mild solution to the problem (1). Also, in Section 3, we apply the Leray-Schauder alternative theorem to prove that problem (1) has at least one mild solution.

\section{Preliminaries}

In this section, we present some definitions and properties of fractional calculus which will be used throughout this paper. 
Definition 2.1. [11] The Laplace transform of a function $f$ is denoted and defined by:

$$
\mathcal{L}\{f(t)\}(s)=F(s)=\int_{0}^{\infty} f(t) e^{-s t} d t \text { for } s>0 .
$$

In addition, if $F(s)=\mathcal{L}\{f(t)\}(s)$ and $G(s)=\mathcal{L}\{g(t)\}(s)$, then

$$
\mathcal{L}\left\{\int_{0}^{t} f(t-\tau) g(\tau) d \tau\right\}(s)=F(s) G(s) .
$$

Definition 2.2. [5] Let $f:[0, \infty) \longrightarrow \mathbb{R}$ be a real-valued function and $\psi$ be a non-negative increasing function such that $\psi(0)=0$. Then the Laplace transform of $f$ with respect to $\psi$ is defined by

$$
\mathcal{L}_{\psi}\{f(t)\}(s)=F(s)=\int_{0}^{\infty} f(t) e^{-s \psi(t)} \psi^{\prime}(t) d t \text { for all } s \in \mathbb{C} .
$$

Definition 2.3. [23] Let $(a, b),(-\infty \leq a<b \leq \infty)$ be a finite or infinite interval of the real line $\mathbb{R}$ and $\alpha>0$. Also, let $\psi(x)$ be an increasing and positive monotone function on $(a, b]$, having a continuous derivative $\psi(x)$ on $(a, b)$. Then the left-sided fractional integral of a function $f$ with respect to another function $\psi$ on $[a, b]$ is defined by

$$
I_{a^{+}}^{\alpha ; \psi} f(x)=\frac{1}{\Gamma(\alpha)} \int_{a}^{x} \psi^{\prime}(t)(\psi(x)-\psi(t))^{\alpha-1} f(t) d t
$$

Definition 2.4. [23] Let $\psi^{\prime}(x) \neq 0,(-\infty \leq a<b \leq \infty)$ and $\alpha>0, n \in \mathbb{N}$. The Left-sided Riemann- Liouville derivative of a function $f$ with respect to $\psi$ of order $\alpha$ correspondent to the Riemann-Liouville, is defined by

$$
\begin{aligned}
D_{a^{+}}^{\alpha ; \psi} f(x) & =\left(\frac{1}{\psi^{\prime}(x)} \frac{d}{d x}\right)^{n} I^{n-\alpha ; \psi} f(x) \\
& =\frac{1}{\Gamma(n-\alpha)}\left(\frac{1}{\psi^{\prime}(x)} \frac{d}{d x}\right)^{n} \int_{a}^{x} \psi^{\prime}(t)(\psi(x)-\psi(t))^{n-\alpha-1} f(t) d t .
\end{aligned}
$$

Definition 2.5. [23] Let $\alpha>0, n \in \mathbb{N}, I=[a, b]$ is the interval $(-\infty \leq a<b \leq \infty) . f, \psi \in C^{n}([a, b], \mathbb{R})$ two functions such that $\psi(x)$ is increasing and $\psi^{\prime}(x) \neq 0$ for all $x \in I$. The left-sided $\psi$-Caputo fractional derivative of $f$ of order $\alpha$ is denoted and defined as follow:

$$
{ }^{C} D_{a^{+}}^{\alpha ; \psi} f(x)=I^{n-\alpha ; \psi}\left(\frac{1}{\psi^{\prime}(x)} \frac{d}{d x}\right)^{n} f(x) .
$$

Definition 2.6. [23] Let $n-1<\alpha<n$ with $n \in \mathbb{N}, I=[a, b]$ is the interval such that $(-\infty \leq a<b \leq \infty)$ and, $f, \psi \in C^{n}([a, b], \mathbb{R})$ two functions such that $\psi$ is increasing and $\psi^{\prime}(x) \neq 0$, for all $x \in I$. The left-sided and right-sided $\psi$-Hilfer fractional derivative ${ }^{H} \mathbb{D}^{\alpha, \beta ; \psi}($.$) of function of order \alpha$ and type $0 \leq \beta \leq 1$, are defined respectively by

$$
\begin{aligned}
& { }^{H} \mathbb{D}_{a+}^{\alpha, \beta ; \psi} f(x)=I_{a+}^{\beta(n-\alpha) ; \psi}\left(\frac{1}{\psi^{\prime}(x)} \frac{d}{d x}\right)^{n} I_{a+}^{(1-\beta)(n-\alpha) ; \psi} f(x) \\
& H_{\mathbb{D}_{b-}^{\alpha}}^{\alpha, \beta ; \psi} f(x)=I_{b-}^{\beta(n-\alpha) ; \psi}\left(-\frac{1}{\psi^{\prime}(x)} \frac{d}{d x}\right)^{n} I_{b-}^{(1-\beta)(n-\alpha) ; \psi} f(x) .
\end{aligned}
$$

Definition 2.7. [9] The space $\mathcal{P}$ is a linear space of functions from $(-\infty, 0]$ into $\mathbb{X}$ endowed with a seminorm $\|.\|_{\mathcal{P}}$. The space $\mathcal{P}$ is called an admissible phase space if $\mathcal{P}$ satisfies the following fundamental axioms. If a function $v:(-\infty, T+\delta] \longrightarrow \mathbb{X}$ is such that $\left.v\right|_{[\delta, T+\delta]} \in C([\delta, T+\delta] ; \mathbb{X})$ and $t \in[\delta, T+\delta]$, then 1) $v_{t} \in \mathcal{P}$ for $t \in[\delta, T+\delta]$; 
2)The function $t \mapsto v_{t}$ is continuous on $[\delta, T+\delta]$;

3)There exist a continuous function $\mu_{1}(t)>0$ and a locally bounded function $\mu_{2}(t)>0$ from $[0, \infty) \longrightarrow[0, \infty)$ for $t \geq 0$ which are independent of $v$ such that:

$$
\left|v_{t}\right|_{\mathcal{P}} \leq \mu_{1}(t-\delta) \sup _{\delta \leq s \leq t}\|v(s)\|+\mu_{2}(t-\delta)\left|v_{\delta}\right|_{\mathcal{P}}
$$

4) $\|x(t)\| \leq H\left\|x_{t}\right\|_{\mathcal{P}}$ which is equivalent to $\|\phi(0)\| \leq H\|\phi\|_{\mathcal{P}}$, for all $\phi \in \mathcal{P}$.

Theorem 2.1. [23] If $f \in C^{n}[a, b], n-1<\alpha<n$ and $0 \leq \beta \leq 1, \gamma=\alpha+\beta(n-\alpha)$, then

$$
\begin{aligned}
& I_{a+}^{\alpha ; \psi^{H}} \mathbb{D}_{a+}^{\alpha, \beta ; \psi} f(x)=f(x)-\sum_{k=1}^{n} \frac{(\psi(x)-\psi(a))^{\gamma-k}}{\Gamma(\gamma-k+1)} f_{\psi}^{[n-k]} I_{a+}^{(1-\beta)(n-\alpha) ; \psi} f(a) \\
& I_{b-}^{\alpha ; \psi^{H}} \mathbb{D}_{b-}^{\alpha, \beta ; \psi} f(x)=f(x)-\sum_{k=1}^{n} \frac{(-1)^{k}(\psi(b)-\psi(x))^{\gamma-k}}{\Gamma(\gamma-k+1)} f_{\psi}^{[n-k]} I_{b-}^{(1-\beta)(n-\alpha) ; \psi} f(b) .
\end{aligned}
$$

Theorem 2.2. [23] Let $f \in C^{1}[a, b], \alpha>0$ and $0 \leq \beta \leq 1$, then

$$
\begin{aligned}
& H_{\mathbb{D}_{a+}^{\alpha, \beta ; \psi}}^{\alpha ; I^{+}} f(x)=f(x) \\
& { }_{\mathbb{D}_{b-}^{\alpha, \beta ; \psi}}^{\alpha ;} I_{b-}^{\alpha ; \psi} f(x)=f(x) .
\end{aligned}
$$

Theorem 2.3. [5] Let $\mu>0$ and let $f$ be a function of $\psi$-exponential order, piecewise continuous over each finite interval $[0, T]$. Then

$$
\mathcal{L}_{\psi}\left\{{ }_{0}{ }^{\mu}{ }_{\psi(t)}^{\mu} f\right\}(s)=s^{-\mu} \mathcal{L}_{\psi}\{f(t)\} .
$$

Remark 2. The Laplace transform of the one-sided stable probability density

$$
\rho_{\alpha}(\eta)=\frac{1}{\pi} \sum_{n=1}^{\infty}(-1)^{n-1} \eta^{-\alpha n-1} \frac{\Gamma(\alpha n+1)}{n !} \sin (n \pi \alpha), \quad \eta \in(0, \infty)
$$

is given by

$$
\int_{0}^{\infty} e^{-s \eta} \rho_{\alpha}(\eta) d \eta=e^{-s^{\alpha}}, \text { where } 0<\alpha \leq 1 \text { and } s>0 .
$$

Furthermore, for any $0 \leq \delta \leq 1$, we have (see [21]):

$$
\int_{0}^{\infty} \frac{1}{\eta^{\delta}} \rho_{\alpha}(\eta) d \eta=\frac{\Gamma\left(1+\frac{\delta}{\alpha}\right)}{\Gamma(1+\delta)} .
$$

For more details on the above preliminaries, we refer to [4, 5, 12, 17, 23].

\section{Existence and uniqueness of the mild solution to the Cauchy problem (1).}

We first recall the Banach fixed point principle [8] which we are going to use in this section. Then, we obtain the Volterra integral equivalent equation and mild solution to (1). At the end, we prove the existence and uniqueness of the mild solution based on Banach fixed point principle and the Leray-Schauder alternative theorem. 
Lemma 3.1. Assume $(Z, d)$ to be a non-empty complete metric space, let $0 \leq S<1$ and let the mapping $F$ : $Z \longrightarrow Z$ satisfy the inequality

$$
d(F m, F n) \leq S d(m, n) \text { for every } m, n \in Z \text {. }
$$

Then, $F$ has a uniquely determined fixed point $z^{\star}$. Furthermore, for any $z_{0} \in Z$, the sequence $\left(F^{j} z_{0}\right)_{j=1}^{\infty}$ converges to this fixed point $z^{\star}$.

Proof. The proof of this lemma can be found in [8].

Lemma 3.2. If we assume that $h\left(0, x_{0}\right)=\phi(0)=0$, the Cauchy problem (1) is equivalent to the Volterra integral equation

$$
x(t)= \begin{cases}\frac{\phi(0)-h\left(0, x_{0}\right)}{\Gamma(\gamma) \Gamma(2-\gamma)}+h\left(t, x_{t}\right)+\frac{A}{\Gamma(\alpha)} \int_{0}^{t}(\psi(t)-\psi(s))^{\alpha-1} \psi^{\prime}(s) x(s) d s \\ +\frac{1}{\Gamma(\alpha)} \int_{0}^{t}(\psi(t)-\psi(s))^{\alpha-1} \psi^{\prime}(s) f\left(s, x(s), x_{s}\right) d s, & \text { for } t \in[0, b] \\ \phi(t), & \text { for } t \in(-\infty, 0]\end{cases}
$$

Proof.

Let us assume that $x \in \mathcal{C}$ satisfies the Cauchy problem $(1)$ and $h\left(0, x_{0}\right)=\phi(0)=0$. Then, we prove that (15) holds.

Applying the operator $I_{0^{+}}^{\alpha ; \psi}$ to both sides of the first equation of (1), we have:

$$
I_{0^{+}}^{\alpha ; \psi H} \mathbb{D}_{0^{+}}^{\alpha, \beta ; \psi}\left[x(t)-h\left(t, x_{t}\right)\right]=A I_{0^{+}}^{\alpha ; \psi} x(t)+I_{0^{+}}^{\alpha ; \psi} f\left(t, x(t), x_{t}\right)
$$

By properties of $\psi$-Hilfer fractional derivative and using (10), we obtain the left hand side of (16) as:

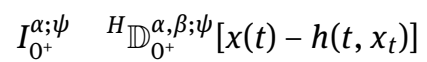

$$
\begin{aligned}
& =\quad x(t)-h\left(t, x_{t}\right)-\frac{(\psi(t)-\psi(0))^{\gamma-1}}{\Gamma(\gamma)} I_{0^{+}}^{(1-\beta)(1-\alpha) ; \psi}\left[x(0)-h\left(0, x_{0}\right)\right] \\
& =\quad x(t)-h\left(t, x_{t}\right)-\frac{(\psi(t)-\psi(0))^{\gamma-1}}{\Gamma(\gamma)} \frac{1}{\Gamma(1-\gamma)} \int_{0}^{t} \psi(s)(\psi(t)-\psi(s))^{-\gamma} \\
& {\left[x(0)-h\left(0, x_{0}\right)\right] d s} \\
& =\quad x(t)-h\left(t, x_{t}\right)-\frac{\phi(0)-h\left(0, x_{0}\right)}{\Gamma(\gamma) \Gamma(2-\gamma)} .
\end{aligned}
$$

In addition, on the right-hand side of (16) we have:

$$
I_{0^{+}}^{\alpha ; \psi} \chi(t)=\frac{1}{\Gamma(\alpha)} \int_{0}^{t}(\psi(t)-\psi(s))^{\alpha-1} \psi^{\prime}(s) \chi(s) d s .
$$

and

$$
I_{0^{+}}^{\alpha ; \psi} f\left(t, x(t), x_{t}\right)=\frac{1}{\Gamma(\alpha)} \int_{0}^{t}(\psi(t)-\psi(s))^{\alpha-1} \psi^{\prime}(s) f\left(s, x(s), x_{s}\right) d s .
$$

Substituting (17), (18) and (19) into (16), we obtain the Volterra integral equation (15).

Conversely, let us assume that $x \in \mathcal{C}$ satisfies the Volterra integral equation (15) and $h\left(0, x_{0}\right)=\phi(0)=0$. Then, we prove that the Cauchy problem (1) holds. 
The first equation of (15) can also be written as

$$
x(t)=h\left(t, x_{t}\right)+\frac{\phi(0)-h\left(0, x_{0}\right)}{\Gamma(\gamma) \Gamma(2-\gamma)}+A I_{0^{+}}^{\alpha ; \psi} x(t)+I_{0^{+}}^{\alpha ; \psi} f\left(t, x(t), x_{t}\right) .
$$

Applying the operator ${ }^{H} \mathbb{D}_{0^{+}}^{\alpha, \beta ; \psi}$ on both sides of the equality (20) while taking into account (11), we have:

$$
\begin{aligned}
{ }^{H} \mathbb{D}_{0^{+}}^{\alpha, \beta ; \psi} x(t) & ={ }^{H} \mathbb{D}_{0^{+}}^{\alpha, \beta ; \psi} h\left(t, x_{t}\right)+{ }^{H} \mathbb{D}_{0^{+}}^{\alpha, \beta ; \psi} \frac{\phi(0)-h\left(0, x_{0}\right)}{\Gamma(\gamma) \Gamma(2-\gamma)} \\
+ & A^{H} \mathbb{D}_{0^{+}}^{\alpha, \beta ; \psi}{ }_{0} I_{t}^{\alpha} x(t)+{ }^{H} \mathbb{D}_{0^{+}}^{\alpha, \beta ; \psi}{ }_{0} I_{t}^{\alpha} f\left(t, x(t), x_{t}\right) .
\end{aligned}
$$

Therefore, we have the following estimate:

$$
{ }^{H} \mathbb{D}_{0^{+}}^{\alpha, \beta ; \psi}\left[x(t)-h\left(t, x_{t}\right)\right]=A x(t)+f\left(t, x(t), x_{t}\right) .
$$

Since, ${ }^{H} \mathbb{D}_{0^{+}}^{\alpha, \beta ; \psi} f(x)=0$ where $f(x)$ is constant and $h\left(0, x_{0}\right)=\phi(0)=0$.

Hence, combining (22) and $x(t)=\phi(t)$ where $t \in(-\infty, 0]$, we conclude that if $x \in \mathcal{C}$ satisfies the Volterra integral equation (15), then $x$ is solution to the Cauchy problem (1). This ends the proof of Lemma 3.2.

Lemma 3.3. If the first equation of (15) holds, then we have the following integral equation:

$$
\begin{aligned}
x(t) & =Q_{\psi}^{\alpha}(t) h_{0}+h\left(t, x_{t}\right)+\int_{0}^{t}(\psi(t)-\psi(s))^{\alpha-1} A R_{\psi}^{\alpha}(t-s) h\left(s, x_{s}\right) \psi^{\prime}(s) d s \\
& +\int_{0}^{t}(\psi(t)-\psi(s))^{\alpha-1} R_{\psi}^{\alpha}(t-s) f\left(s, x(s), x_{s}\right) \psi^{\prime}(s) d s \text { for } t \in[0, b]
\end{aligned}
$$

where $h_{0}$ and the operators $\left\{Q_{\psi}^{\alpha}(t)\right\}_{t \in[0, b]}$ and $\left\{R_{\psi}^{\alpha}(t)\right\}_{t \in[0, b]}$ are defined by

$$
\begin{gathered}
h_{0}=\frac{\phi(0)-h\left(0, x_{0}\right)}{\Gamma(\gamma) \Gamma(2-\gamma)} \\
Q_{\psi}^{\alpha}(t) x=\int_{0}^{\infty} \zeta_{\alpha}(\eta) T\left((\psi(t)-\psi(0))^{\alpha} \eta\right) x d \eta \quad \forall x \in \mathbb{X}
\end{gathered}
$$

and

$$
R_{\psi}^{\alpha}(t) x=\alpha \int_{0}^{\infty} \eta \zeta_{\alpha}(\eta) T\left((\psi(t)-\psi(0))^{\alpha} \eta\right) x d \eta \quad \forall x \in \mathbb{X}
$$

where

$$
\zeta_{\alpha}(\eta)=\frac{1}{\alpha} \eta^{-\frac{1}{\alpha}-1} \rho_{\alpha}\left(\eta^{-\frac{1}{\alpha}}\right)
$$

is the probability density function defined on $(0, \infty)$, that is, $\zeta_{\alpha}(\eta) \geq 0$ for $\eta \in(0, \infty)$ and $\int_{0}^{\infty} \zeta_{\alpha}(\eta) d \eta=1$. 
Proof. Let $p>0$ and $h_{0}=\frac{\phi(0)-h\left(0, x_{0}\right)}{\Gamma(\gamma) \Gamma(2-\gamma)}$. Applying the generalized Laplace transform on both sides of the first equation of (15), we have:

$$
\begin{aligned}
\mathcal{L}_{\psi}\{x(t)\}(p)= & h_{0} \mathcal{L}_{\psi}\{1\}(p)+\mathcal{L}_{\psi}\left\{h\left(t, x_{t}\right)\right\}(p) \\
& +\frac{A}{\Gamma(\alpha)} \mathcal{L}_{\psi}\left\{\int_{0}^{t}(\psi(t)-\psi(s))^{\alpha-1} \psi^{\prime}(s) x(s) d s\right\}(p) \\
& +\frac{1}{\Gamma(\alpha)} \mathcal{L}_{\psi}\left\{\int_{0}^{t}(\psi(t)-\psi(s))^{\alpha-1} \psi^{\prime}(s) f\left(s, x(s), x_{s}\right) d s\right\}(p)
\end{aligned}
$$

By using (3), (4) and (12) and considering $X(p)=\mathcal{L}_{\psi}\{x(t)\}(p), H(p)=\mathcal{L}_{\psi}\left\{h\left(t, x_{t}\right)\right\}(p)$ and $G(p)=$ $\mathcal{L}_{\psi}\left\{f\left(t, x(t), x_{t}\right)\right\}(p)$ as follows:

$$
\begin{aligned}
& X(p)=\int_{0}^{\infty} e^{-p(\psi(\tau)-\psi(0))} \psi^{\prime}(\tau) x(\tau) d \tau \\
& H(p)=\int_{0}^{\infty} e^{-p(\psi(\tau)-\psi(0))} \psi^{\prime}(\tau) h\left(\tau, x_{\tau}\right) d \tau \\
& G(p)=\int_{0}^{\infty} e^{-p(\psi(\tau)-\psi(0))} \psi^{\prime}(\tau) f\left(\tau, x(\tau), x_{\tau}\right) d \tau
\end{aligned}
$$

We obtain:

$$
\begin{aligned}
X(p)= & p^{\alpha-1}\left(p^{\alpha} I-A\right)^{-1} h_{0}+p^{\alpha}\left(p^{\alpha} I-A\right)^{-1} H(p)+\left(p^{\alpha} I-A\right)^{-1} G(p) \\
= & p^{\alpha-1} \mathcal{L}\{T(\tau)\}\left(p^{\alpha}\right) h_{0}+p^{\alpha} \mathcal{L}\{T(\tau)\}\left(p^{\alpha}\right) H(p)+\mathcal{L}\{T(\tau)\}\left(p^{\alpha}\right) G(p) \\
= & p^{\alpha-1} \int_{0}^{\infty} e^{-p^{\alpha} \tau} T(\tau) h_{0} d \tau+p^{\alpha} \int_{0}^{\infty} e^{-p^{\alpha} \tau} T(\tau) H(p) d \tau \\
& +\int_{0}^{\infty} e^{-p^{\alpha} \tau} T(\tau) G(p) d \tau .
\end{aligned}
$$

Now we consider the following change of variable: $\tau=\hat{t}^{\alpha}$ and $d \tau=\alpha \hat{t}^{\alpha-1} d \hat{t}$.

Equation (30) becomes:

$$
\begin{aligned}
X(p)= & p^{\alpha-1} \int_{0}^{\infty} \alpha \hat{t}^{\alpha-1} e^{-(p \hat{t})^{\alpha}} T\left(\hat{t}^{\alpha}\right) h_{0} d \hat{t}+p^{\alpha} \int_{0}^{\infty} \alpha \hat{t}^{\alpha-1} e^{-(p \hat{t})^{\alpha}} T\left(\hat{t}^{\alpha}\right) H(p) d \hat{t} \\
& +\int_{0}^{\infty} \alpha \hat{t}^{\alpha-1} e^{-(p \hat{t})^{\alpha}} T\left(\hat{t}^{\alpha}\right) G(p) d \hat{t} \\
= & \alpha \int_{0}^{\infty}(p \hat{t})^{\alpha-1} e^{-(p \hat{t})^{\alpha}} T\left(\hat{t}^{\alpha}\right) h_{0} d \hat{t}+\alpha \int_{0}^{\infty} p^{\alpha} \hat{t}^{\alpha-1} e^{-(p \hat{t})^{\alpha}} T\left(\hat{t}^{\alpha}\right) H(p) d \hat{t} \\
& +\alpha \int_{0}^{\infty} \hat{t}^{\alpha-1} e^{-(p \hat{t})^{\alpha}} T\left(\hat{t}^{\alpha}\right) G(p) d \hat{t} .
\end{aligned}
$$


Take $\hat{t}=\psi(t)-\psi(0)$. Then, we get:

$$
\begin{aligned}
X(p)= & \alpha \int_{0}^{\infty} p^{\alpha-1}(\psi(t)-\psi(0))^{\alpha-1} e^{-(p(\psi(t)-\psi(0)))^{\alpha}} T\left((\psi(t)-\psi(0))^{\alpha}\right) h_{0} \psi^{\prime}(t) d t \\
& +\alpha \int_{0}^{\infty} p^{\alpha}(\psi(t)-\psi(0))^{\alpha-1} e^{-(p(\psi(t)-\psi(0)))^{\alpha}} T\left((\psi(t)-\psi(0))^{\alpha}\right) H(p) \psi^{\prime}(t) d t \\
& +\alpha \int_{0}^{\infty}(\psi(t)-\psi(0))^{\alpha-1} e^{-(p(\psi(t)-\psi(0)))^{\alpha}} T\left((\psi(t)-\psi(0))^{\alpha}\right) G(p) \psi^{\prime}(t) d t \\
= & \alpha \int_{0}^{\infty} p^{\alpha-1}(\psi(t)-\psi(0))^{\alpha-1} e^{-(p(\psi(t)-\psi(0)))^{\alpha}} T\left((\psi(t)-\psi(0))^{\alpha}\right) h_{0} \psi^{\prime}(t) d t \\
& +\alpha \int_{0}^{\infty} \int_{0}^{\infty} p^{\alpha}(\psi(t)-\psi(0))^{\alpha-1} e^{-(p(\psi(t)-\psi(0)))^{\alpha}} T\left((\psi(t)-\psi(0))^{\alpha}\right) \\
& e^{-(p(\psi(s)-\psi(0)))} h\left(s, x_{s}\right) \psi^{\prime}(s) \psi^{\prime}(t) d s d t \\
& +\alpha \int_{0}^{\infty} \int_{0}^{\infty}(\psi(t)-\psi(0))^{\alpha-1} e^{-(p(\psi(t)-\psi(0)))^{\alpha}} T\left((\psi(t)-\psi(0))^{\alpha}\right) \\
& e^{-(p(\psi(s)-\psi(0)))} f\left(s, x(s), x_{s}\right) \psi^{\prime}(s) \psi^{\prime}(t) d s d t .
\end{aligned}
$$

Now, equation (32) becomes:

$$
\begin{aligned}
X(p)= & \int_{0}^{\infty}-\frac{1}{p} T\left((\psi(t)-\psi(0))^{\alpha}\right) h_{0}\left(\frac{d}{d t}\left(e^{-(p(\psi(t)-\psi(0)))^{\alpha}}\right)\right) d t \\
& +\int_{0}^{\infty}\left[-T\left((\psi(t)-\psi(0))^{\alpha}\right) e^{-p(\psi(s)-\psi(0))} h\left(s, x_{s}\right) \psi^{\prime}(s) d s\right] \\
& \frac{d}{d t} e^{-p(\psi(t)-\psi(0))^{\alpha}} d t+\int_{0}^{\infty} \int_{0}^{\infty} \alpha(\psi(t)-\psi(0))^{\alpha-1} e^{-(p(\psi(t)-\psi(0)))^{\alpha}} \\
& T\left((\psi(t)-\psi(0))^{\alpha}\right) e^{-(p(\psi(s)-\psi(0)))} f\left(s, x(s), x_{s}\right) \psi^{\prime}(s) \psi^{\prime}(t) d s d t
\end{aligned}
$$


Taking into account (13) which is the Laplace transform of the one-sided probability density, we obtain:

$$
\begin{aligned}
X(p)= & \int_{0}^{\infty} \int_{0}^{\infty} \sigma \rho_{\alpha}(\sigma) e^{-(p(\psi(t)-\psi(0)) \sigma)} T\left((\psi(t)-\psi(0))^{\alpha}\right) h_{0} \psi^{\prime}(t) d \sigma d t \\
& +\left[e^{-(p(\psi(t)-\psi(0)))^{\alpha}} \int_{0}^{\infty}-T\left((\psi(t)-\psi(0))^{\alpha}\right) e^{-(p(\psi(s)-\psi(0)))} h\left(s, x_{s}\right) \psi^{\prime}(s) d s\right]_{t=0}^{\infty} \\
& +\int_{0}^{\infty} \int_{0}^{\infty} \alpha(\psi(t)-\psi(0))^{\alpha-1} e^{-(p(\psi(t)-\psi(0)))^{\alpha}} \\
& A T\left((\psi(t)-\psi(0))^{\alpha}\right) e^{-(p(\psi(s)-\psi(0)))} h\left(s, x_{s}\right) \psi^{\prime}(s) \psi^{\prime}(t) d s d t \\
& +\int_{0}^{\infty} \int_{0}^{\infty} \int_{0}^{\infty} \alpha(\psi(t)-\psi(0))^{\alpha-1} e^{-(p(\psi(t)-\psi(0)) \sigma)} \rho_{\alpha}(\sigma) \\
& T\left((\psi(t)-\psi(0))^{\alpha}\right) e^{-(p(\psi(s)-\psi(0)))} f\left(s, x(s), x_{s}\right) \psi^{\prime}(s) \psi^{\prime}(t) d \sigma d s d t .
\end{aligned}
$$

Now we consider the change of variable $\psi(t)-\psi(0)=\frac{\psi(\theta)-\psi(0)}{\sigma} \rightarrow \psi^{\prime}(\theta) d \theta=\sigma \psi^{\prime}(t) d t$.Then, equation (34) becomes

$$
\begin{aligned}
X(p)= & \int_{0}^{\infty} \int_{0}^{\infty} \rho_{\alpha}(\sigma) e^{-(p(\psi(\theta)-\psi(0)))} T\left(\frac{(\psi(\theta)-\psi(0))^{\alpha}}{\sigma^{\alpha}}\right) h_{0} \psi^{\prime}(\theta) d \sigma d \theta \\
& +\int_{0}^{\infty} e^{-(p(\psi(t)-\psi(0)))}\left[h\left(t, x_{t}\right)+\alpha \int_{0}^{\infty} \int_{0}^{\infty} A T\left(\frac{(\psi(\theta)-\psi(0))^{\alpha}}{\sigma^{\alpha}}\right)\right. \\
& \left.\left(\frac{(\psi(\theta)-\psi(0))^{\alpha-1}}{\sigma^{\alpha}}\right) \rho_{\alpha}(\sigma) h\left(s, x_{S}\right) \psi^{\prime}(s) \psi^{\prime}(\theta) d \sigma d \theta\right] \psi^{\prime}(t) d t \\
& +\alpha \int_{0}^{\infty} \int_{0}^{\infty} \int_{0}^{t} e^{-(p(\psi(\theta)+\psi(s)-2 \psi(0)))}\left(\frac{(\psi(\theta)-\psi(0))^{\alpha-1}}{\sigma^{\alpha}}\right) \rho_{\alpha}(\sigma) \\
& T\left(\frac{(\psi(\theta)-\psi(0))^{\alpha}}{\sigma^{\alpha}}\right) f\left(s, x(s), x_{s}\right) \psi^{\prime}(s) \psi^{\prime}(\theta) d \sigma d s d \theta .
\end{aligned}
$$

Considering the new change of variable $\psi(\theta)+\psi(s)-2 \psi(0)=\psi(\tau)-\psi(0) \rightarrow \psi(\tau)-\psi(s)=\psi(\theta)-\psi(0)$, we have:

$$
\left\{\begin{array}{l}
\theta \rightarrow 0 \quad \Rightarrow \tau \rightarrow s \\
\theta \rightarrow \infty \quad \Rightarrow \tau \rightarrow \infty
\end{array},\left\{\begin{array}{l}
0<s<\infty \\
s<\tau<\infty
\end{array} \Rightarrow 0<s<\tau\right.\right.
$$

and

$$
\left\{\begin{array}{l}
0<s<\infty \\
s<\tau<\infty
\end{array} \Longrightarrow 0<\tau<\infty\right.
$$

Then, (35) becomes: 


$$
\begin{aligned}
& X(p)=\int_{0}^{\infty} e^{-(p(\psi(\theta)-\psi(0)))}\left[\int_{0}^{\infty} \rho_{\alpha}(\sigma) T\left(\frac{(\psi(\theta)-\psi(0))^{\alpha}}{\sigma^{\alpha}}\right) h_{0} d \sigma\right] \psi^{\prime}(\theta) d \theta \\
& +\int_{0}^{\infty} e^{-(p(\psi(\tau)-\psi(0)))}\left[h\left(\tau, x_{\tau}\right)+\alpha \int_{0}^{\infty} \int_{0}^{\infty} A T\left(\frac{(\psi(\theta)-\psi(0))^{\alpha}}{\sigma^{\alpha}}\right)\right. \\
& \left(\frac{(\psi(\theta)-\psi(0))^{\alpha-1}}{\sigma^{\alpha}}\right) \rho_{\alpha}(\sigma) h\left(\psi^{-1}(\psi(\tau)-\psi(\theta)+\psi(0),\right. \\
& \left.\left.\chi_{\psi^{-1}(\psi(\tau)-\psi(\theta)+\psi(0)}\right) \psi^{\prime}(\theta) d \sigma d \theta\right] \psi^{\prime}(\tau) d \tau \\
& +\alpha \int_{0}^{\infty} \int_{0}^{\infty} \int_{0}^{\infty} e^{-(p(\psi(\tau)-\psi(0)))}\left(\frac{(\psi(\theta)-\psi(0))^{\alpha-1}}{\sigma^{\alpha}}\right) \rho_{\alpha}(\sigma) \\
& T\left(\frac{(\psi(\theta)-\psi(0))^{\alpha}}{\sigma^{\alpha}}\right) f\left(\psi ^ { - 1 } \left(\psi(\tau)-\psi(\theta)+\psi(0), x\left(\psi^{-1}(\psi(\tau)-\psi(\theta)\right.\right.\right. \\
& \left.\left.+\psi(0), x_{\psi^{-1}(\psi(\tau)-\psi(\theta)+\psi(0)}\right)\right) \psi^{\prime}(\tau) \psi^{\prime}(\theta) d \sigma d \tau d \theta \\
& X(p)=\int_{0}^{\infty} e^{-(p(\psi(\tau)-\psi(0)))}\left[\int_{0}^{\infty} \rho_{\alpha}(\sigma) T\left(\frac{(\psi(\tau)-\psi(0))^{\alpha}}{\sigma^{\alpha}}\right) h_{0} d \sigma+h\left(\tau, x_{\tau}\right)\right. \\
& +\alpha \int_{0}^{\tau} \int_{0}^{\infty} A T\left(\frac{(\psi(\tau)-\psi(s))^{\alpha}}{\sigma^{\alpha}}\right)\left(\frac{(\psi(\tau)-\psi(s))^{\alpha-1}}{\sigma^{\alpha}}\right) \rho_{\alpha}(\sigma) h\left(s, x_{s}\right) \\
& \psi^{\prime}(s) d \sigma d s+\alpha \int_{0}^{\tau} \int_{0}^{\infty}\left(\frac{(\psi(\tau)-\psi(s))^{\alpha-1}}{\sigma^{\alpha}}\right) \rho_{\alpha}(\sigma) T\left(\frac{(\psi(\tau)-\psi(s))^{\alpha}}{\sigma^{\alpha}}\right) \\
& \left.f\left(s, x(s), x_{s}\right) \psi^{\prime}(s) d \sigma d s\right] \psi^{\prime}(\tau) d \tau
\end{aligned}
$$

Applying the inverse Laplace transform on (36), we deduce that

$$
\begin{aligned}
x(t)= & \int_{0}^{\infty} \rho_{\alpha}(\sigma) T\left(\frac{(\psi(t)-\psi(0))^{\alpha}}{\sigma^{\alpha}}\right) h_{0} d \sigma+h\left(t, x_{t}\right) \\
& \alpha \int_{0}^{\infty} \int_{0}^{t} A T\left(\frac{(\psi(t)-\psi(s))^{\alpha}}{\sigma^{\alpha}}\right)\left(\frac{(\psi(t)-\psi(s))^{\alpha-1}}{\sigma^{\alpha}}\right) \rho_{\alpha}(\sigma) \\
& h\left(s, x_{s}\right) \psi^{\prime}(s) d s d \sigma+\alpha \int_{0}^{\infty} \int_{0}^{t}\left(\frac{(\psi(t)-\psi(s))^{\alpha-1}}{\sigma^{\alpha}}\right) \rho_{\alpha}(\sigma) \\
& T\left(\frac{(\psi(t)-\psi(s))^{\alpha}}{\sigma^{\alpha}}\right) f\left(s, x(s), x_{s}\right) \psi^{\prime}(s) d s d \sigma
\end{aligned}
$$

Now we consider the following change of variable:

$$
\frac{1}{\sigma^{\alpha}}=\eta \Rightarrow \sigma=\eta^{-\frac{1}{\alpha}} \text { and } d \sigma=-\frac{1}{\alpha} \eta^{-\frac{1}{\alpha}-1} d \eta .
$$


Equation (37) becomes

$$
\begin{aligned}
x(t)= & \int_{0}^{\infty} T\left((\psi(t)-\psi(0))^{\alpha} \eta\right)\left(\frac{1}{\alpha} \eta^{-\frac{1}{\alpha}-1} \rho_{\alpha}\left(\eta^{-\frac{1}{\alpha}}\right)\right) h_{0} d \eta+h\left(x, x_{t}\right) \\
& +\alpha \int_{0}^{\infty} \int_{0}^{t} \eta(\psi(t)-\psi(0))^{\alpha-1} A T\left((\psi(t)-\psi(0))^{\alpha} \eta\right) \\
& \left(\frac{1}{\alpha} \eta^{-\frac{1}{\alpha}-1} \rho_{\alpha}\left(\eta^{-\frac{1}{\alpha}}\right)\right) h\left(s, x_{S}\right) \psi^{\prime}(s) d s d \eta \\
& +\alpha \int_{0}^{\infty} \int_{0}^{t} \eta(\psi(t)-\psi(0))^{\alpha-1} T\left((\psi(t)-\psi(0))^{\alpha} \eta\right) \\
& \left(\frac{1}{\alpha} \eta^{-\frac{1}{\alpha}-1} \rho_{\alpha}\left(\eta^{-\frac{1}{\alpha}}\right)\right) f\left(s, x(s), x_{s}\right) \psi^{\prime}(s) d s d \eta
\end{aligned}
$$

Thus, we have:

$$
\begin{aligned}
X(t)= & \int_{0}^{\infty} T\left((\psi(t)-\psi(0))^{\alpha} \eta\right) \zeta_{\alpha}(\eta) h_{0} d \eta+\alpha \int_{0}^{\infty} \int_{0}^{t} \eta(\psi(t)-\psi(s))^{\alpha-1} \\
& A T\left((\psi(t)-\psi(s))^{\alpha} \eta\right) \zeta_{\alpha}(\eta) h\left(s, x_{s}\right) \psi^{\prime}(s) d s d \eta+\alpha \int_{0}^{\infty} \int_{0}^{t} \eta(\psi(t) \\
& -\psi(s))^{\alpha-1} T\left((\psi(t)-\psi(s))^{\alpha} \eta\right) \zeta_{\alpha}(\eta) f\left(s, x(s), x_{s}\right) \psi^{\prime}(s) d s d \eta
\end{aligned}
$$

where

$$
\zeta_{\alpha}(\eta)=\frac{1}{\alpha} \eta^{-\frac{1}{\alpha}-1} \rho_{\alpha}\left(\eta^{-\frac{1}{\alpha}}\right), \quad \eta \in(0, \infty)
$$

Therefore,

$$
\begin{aligned}
x(t) & =Q_{\psi}^{\alpha}(t) h_{0}+h\left(t, x_{t}\right)+\int_{0}^{t}(\psi(t)-\psi(s))^{\alpha-1} A R_{\psi}^{\alpha}(t-s) h\left(s, x_{s}\right) \psi^{\prime}(s) d s \\
& +\int_{0}^{t}(\psi(t)-\psi(s))^{\alpha-1} R_{\psi}^{\alpha}(t-s) f\left(s, x(s), x_{s}\right) \psi^{\prime}(s) d s \text { for } t \in[0, b]
\end{aligned}
$$

where $h_{0}$ and the operators $\left\{Q_{\psi}^{\alpha}(t)\right\}_{t \in[0, b]}$ and $\left\{R_{\psi}^{\alpha}(t)\right\}_{t \in[0, b]}$ are defined by (23), (24) and (25), respectively and the proof of Lemma 3.3 is complete.

Motivated by Lemma 3.3, we give the following definition of the mild solution of the Cauchy problem (1).

Definition 3.1. A function $x:(-\infty, b] \longrightarrow X$ is said to be the mild solution to the Cauchy problem (1) if $x(t)=$ $\phi(t)$ for $t \leq 0$ and

$$
\begin{aligned}
x(t) & =Q_{\psi}^{\alpha}(t) h_{0}+h\left(t, x_{t}\right)+\int_{0}^{t}(\psi(t)-\psi(s))^{\alpha-1} A R_{\psi}^{\alpha}(t-s) h\left(s, x_{s}\right) \psi^{\prime}(s) d s \\
& +\int_{0}^{t}(\psi(t)-\psi(s))^{\alpha-1} R_{\psi}^{\alpha}(t-s) f\left(s, x(s), x_{s}\right) \psi^{\prime}(s) d s \text { for } t \in[0, b]
\end{aligned}
$$


where $h_{0}$ and the operators $\left\{Q_{\psi}^{\alpha}(t)\right\}_{t \in[0, b]}$ and $\left\{R_{\psi}^{\alpha}(t)\right\}_{t \in[0, b]}$ are defined by (23), (24) and (25), respectively

Therefore, for $\phi \in \mathcal{P}$ and $x \in \mathcal{C}$, we denote the mild solution $x[\phi]:(-\infty, b] \longrightarrow X$ for problem (1) as:

$$
x[\phi](t)=\left\{\begin{array}{l}
x(t) \text { for } t \in[0, b] \\
\phi(t) \text { for } t \in(-\infty, 0]
\end{array}\right.
$$

Lemma 3.4. For any fixed $t \in[0, b], Q_{\psi}^{\alpha}(t)$ and $R_{\psi}^{\alpha}(t)$ are linear bounded operators.

Proof. For any fixed $t \in[0, b], T(t)$ is a linear operator. So, for any $0 \leq \delta \leq 1$ we have the following:

$$
\begin{aligned}
\int_{0}^{\infty} \eta^{\delta} \zeta_{\alpha}(\eta) d \eta & =\int_{0}^{\infty} \eta^{\delta} \frac{1}{\alpha} \eta^{-\frac{1}{\alpha}-1} \rho_{\alpha}\left(\eta^{-\frac{1}{\alpha}}\right) d \eta \\
& =\int_{0}^{\infty} \frac{1}{\alpha} \eta^{-\frac{1}{\alpha}-1+\delta} \rho_{\alpha}\left(\eta^{-\frac{1}{\alpha}}\right) d \eta .
\end{aligned}
$$

Now we consider the following change of variable:

$$
\sigma=\eta^{-\frac{1}{\alpha}} \Rightarrow \eta=\sigma^{-\alpha} \Rightarrow d \eta=-\alpha \sigma^{-\alpha-1} d \sigma .
$$

So, equation (43) becomes:

$$
\begin{aligned}
\int_{0}^{\infty} \eta^{\delta} \zeta_{\alpha}(\eta) d \eta & =\int_{0}^{\infty} \sigma^{-\alpha \delta} \rho_{\alpha}(\sigma) d \sigma \\
& =\int_{0}^{\infty} \frac{1}{\sigma^{\alpha \delta}} \rho_{\alpha}(\sigma) d \sigma .
\end{aligned}
$$

But $\left\{\begin{array}{l}0 \leq \delta \leq 1 \\ 0<\alpha \leq 1\end{array} \Rightarrow 0 \leq \alpha \delta \leq 1\right.$.

Then, from (14), we deduce that

$$
\int_{0}^{\infty} \eta^{\delta} \zeta_{\alpha}(\eta) d \eta=\frac{\Gamma(1+\delta)}{\Gamma(1+\alpha \delta)} .
$$

In particular, for $\delta=1$, we deduce the following:

$$
\int_{0}^{\infty} \eta \zeta_{\alpha}(\eta) d \eta=\frac{1}{\Gamma(\alpha+1)} .
$$

For any $x \in \mathbb{X}$, by remark 1 we have:

$$
\begin{aligned}
\left\|Q_{\psi}^{\alpha}(t) x\right\| & =\left\|\int_{0}^{\infty} \zeta_{\alpha}(\eta) T\left((\psi(t)-\psi(0))^{\alpha} \eta\right) x d \eta\right\|_{=K} \\
& \leq \underbrace{\sup _{\tau \in[0, \infty)}\|T(\psi(\tau)-\psi(0))\|}_{=1}\|x\| \underbrace{\int_{0}^{\infty} \zeta_{\alpha}(\eta) d \eta}_{=1} \\
& =K\|x\| \\
\Rightarrow \frac{\left\|Q_{\psi}^{\alpha}(t) x\right\|}{\|x\|} & \leq K
\end{aligned}
$$


which implies that

$$
\left\|Q_{\psi}^{\alpha}(t)\right\|_{\mathcal{B}(\mathbb{X})} \leq K
$$

In addition, we have for any $x \in \mathbb{X}$ the following:

$$
\begin{aligned}
\left\|R_{\psi}^{\alpha} x\right\| & =\left\|\alpha \int_{0}^{\infty} \eta \zeta_{\alpha}(\eta) T\left((\psi(t)-\psi(0))^{\alpha} \eta\right) x d \eta\right\|_{=K} \\
& \leq \alpha \underbrace{\sup _{\tau \in[0, \infty)}\|T(\psi(\tau)-\psi(0))\|}_{=\frac{1}{\Gamma(\alpha+1)}}\|x\| \underbrace{\int_{0}^{\infty} \eta \zeta_{\alpha}(\eta) d \eta} \\
& =\frac{\alpha K}{\Gamma(\alpha+1)}\|x\| \\
\Rightarrow \frac{\left\|R_{\psi}^{\alpha} x\right\|}{\|x\|} & \leq \frac{\alpha K}{\Gamma(\alpha+1)}
\end{aligned}
$$

which implies that

$$
\left\|R_{\psi}^{\alpha}\right\|_{\mathcal{B}(\mathbb{X})} \leq \frac{\alpha K}{\Gamma(\alpha+1)} .
$$

From inequalities (46) and (47), we deduce that the operators $\left\{Q_{\psi}^{\alpha}(t)\right\}_{t \in[0, b]}$ and $\left\{R_{\psi}^{\alpha}\right\}_{t \in[0, b]}$ are linear and bounded.

We assume that

$$
\begin{array}{ll}
\left(H_{1}\right): \quad h:[0, b] \times \mathcal{P} \longrightarrow \mathbb{X} \text { is a continuous function and there exists } \\
& N>0, \text { such that for any } t \in I \text { and } x_{t}, x_{t}^{\star} \in \mathcal{P}: \\
& \left\|h\left(t, x_{t}\right)-h\left(t, x_{t}^{\star}\right)\right\| \leq N\left\|x_{t}-x_{t}^{\star}\right\|_{\mathcal{P} .} \\
& f:[0, b] \times \mathbb{X} \times \mathcal{P} \longrightarrow \mathbb{X} \text { is a continuous function and there exists } C_{1}, \\
& C_{2}>0, \text { such that for any } t \in I \text { and } x, x^{\star} \in \mathbb{X} \text { and } y, y^{\star} \in \mathcal{P} \\
\left(H_{2}\right): \quad & \left\|f(t, x, y)-f\left(t, x^{\star}, y^{\star}\right)\right\| \leq C_{1}\left\|x-x^{\star}\right\|+C_{2}\left\|y-y^{\star}\right\|_{\mathcal{P}} .
\end{array}
$$

Theorem 3.1. Under the assumptions $\left(H_{1}\right)-\left(H_{2}\right)$, the Neutral Cauchy problem (1) has a unique mild solution provided that $A$ to be a bounded linear operator and there exists a constant $0<L_{\alpha}<1$ such that:

$$
L_{\alpha}=N \mu_{1}^{\star}+\frac{\|A\| K N \mu_{1}^{\star}}{\Gamma(\gamma+1)} \psi(b)^{\alpha}+\frac{K\left(C_{1}+C_{2} \mu_{1}^{\star}\right)}{\Gamma(\gamma+1)} \psi(b)^{\alpha}
$$

Proof. Consider the operator $N: \mathcal{C} \longrightarrow \mathcal{C}$. We have:

$$
N(x)(t)=\left\{\begin{array}{lr}
Q_{\psi}^{\alpha}(t) h_{0}+h\left(t, x_{t}\right)+\int_{0}^{t}(\psi(t)-\psi(s))^{\alpha-1} A R_{\psi}^{\alpha}(t-s) \\
h\left(s, x_{s}\right) \psi^{\prime}(s) d s+\int_{0}^{t}(\psi(t)-\psi(s))^{\alpha-1} R_{\psi}^{\alpha}(t-s) f(s, x(s), \\
\left.x_{s}\right) \psi^{\prime}(s) d s & \text { for } t \in[0, b] \\
\phi(t) & \text { for } t \in(-\infty, 0]
\end{array}\right.
$$


Let $y():.(-\infty, b] \longrightarrow \mathbb{X}$ be the function as:

$$
y(t)= \begin{cases}0 & \text { for } t \in[0, b] \\ \phi(t) & \text { for } t \in(-\infty, 0]\end{cases}
$$

which we have $y_{0}=\phi(0)$. Then for each $z \in \mathcal{C}([0, b], \mathbb{X})$ with $z(0)=0$, we define the function $Z$ by the following:

$$
z(t)=\left\{\begin{array}{lr}
w(t) & \text { for } t \in[0, b] \\
0 & \text { for } t \in(-\infty, 0]
\end{array}\right.
$$

If $x($.$) verifies the mild solution (42), then by x(t)=y(t)+z(t)$ for $t \in[0, b]$, we have $x_{t}=y_{t}+z_{t}$ for $t \in[0, b]$ and,

$$
\begin{aligned}
w(t)= & Q_{\psi}^{\alpha}(t) h_{0}+h\left(t, y_{t}+z_{t}\right)+\int_{0}^{t}(\psi(t)-\psi(s))^{\alpha-1} A R_{\psi}^{\alpha}(t-s) \\
& h\left(s, y_{s}+z_{s}\right) \psi^{\prime}(s) d s+\int_{0}^{t}(\psi(t)-\psi(s))^{\alpha-1} R_{\psi}^{\alpha}(t-s) \\
& f\left(s, y(s)+z(s), y_{s}+z_{s}\right) \psi^{\prime}(s) d s
\end{aligned}
$$

In Addition, $w_{0}=0$. Let $W_{0}=\left\{w \in C\right.$ such that $\left.w_{0}=0\right\}$. For any $z \in W_{0},\|z\|_{W_{0}}=\sup \|z(t)\|+\left\|z_{0}\right\|_{\mathcal{P}}=$ $\sup _{t \in I}\|z(t)\|$. Therefore, $\left(W_{0},\|\cdot\|_{W_{0}}\right)$ is a Banach space.

We define the operator $G: W_{0} \longrightarrow W_{0}$ by:

$$
\begin{aligned}
G(w)(t)= & Q_{\psi}^{\alpha}(t) h_{0}+h\left(t, y_{t}+z_{t}\right)+\int_{0}^{t}(\psi(t)-\psi(s))^{\alpha-1} A R_{\psi}^{\alpha}(t-s) \\
& h\left(s, y_{s}+z_{s}\right) \psi^{\prime}(s) d s+\int_{0}^{t}(\psi(t)-\psi(s))^{\alpha-1} R_{\psi}^{\alpha}(t-s) \\
& f\left(s, y(s)+z(s), y_{s}+z_{s}\right) \psi^{\prime}(s) d s
\end{aligned}
$$

By assumptions $\left(H_{1}\right)$ and $\left(H_{2}\right)$, we deduce that the operator $G$ is well-defined and the operator $N$ has a unique fixed point if and only if $G$ has a unique fixed point. So we need to prove further that $G$ has a unique fixed point. Consider $z, z^{\star} \in W_{0}$. So, for any $t \in[0, b]$ we obtain: 


$$
\begin{aligned}
\left\|G z(t)-G z^{*}(t)\right\|= & \|\left(h\left(t, y_{t}+z_{t}\right)-h\left(t, y_{t}+z_{t}^{\star}\right)\right)+\int_{0}^{t}(\psi(t)-\psi(s))^{\alpha-1} \\
& A R_{\psi}^{\alpha}(t-s)\left(h\left(s, y_{s}+z_{s}\right)-h\left(s, y_{s}+z_{s}^{\star}\right)\right) \psi^{\prime}(s) d s \\
+ & \int_{0}^{t}(\psi(t)-\psi(s))^{\alpha-1} R_{\psi}^{\alpha}(t-s)\left(f\left(s, y(s)+z(s), y_{s}+z_{s}\right)\right. \\
& \left.-f\left(s, y(s)+z^{*}(s), y_{s}+z_{s}^{\star}\right)\right) \psi^{\prime}(s) d s \| \\
= & \left\|h\left(t, y_{t}+z_{t}\right)-h\left(t, y_{t}+z_{t}^{\star}\right)\right\|+\int_{0}^{t}(\psi(t)-\psi(s))^{\alpha-1} \\
& \|A\|\left\|R_{\psi}^{\alpha}(t-s)\right\|\left\|h\left(s, y_{s}+z_{s}\right)-h\left(s, y_{s}+z_{s}^{*}\right)\right\| \psi^{\prime}(s) d s \\
+ & \int_{0}^{t}(\psi(t)-\psi(s))^{\alpha-1}\left\|R_{\psi}^{\alpha}(t-s)\right\| \| f\left(s, y(s)+z(s), y_{s}+z_{s}\right) \\
& -f\left(s, y(s)+z^{\star}(s), y_{s}+z_{s}^{\star}\right) \| \psi^{\prime}(s) d s
\end{aligned}
$$

By Definition 2.7 related to phase space and assumption $\left(H_{1}\right)$, let $\mu_{1}^{\star}(t)=\sup _{t \in[0, b]} \mu_{1}(t)$. Then, since $z_{0}=0$ we have:

$$
\begin{aligned}
\left\|h\left(t, y_{t}+z_{t}\right)-h\left(t, y_{t}+z_{t}^{\star}\right)\right\| & \leq N\left(\mu_{1}(t) \sup _{s \in[0, b]}\left\|z_{s}-z_{s}^{\star}\right\|+\mu_{2}(t)\left\|z_{0}-z_{0}^{\star}\right\|_{\mathcal{P}}\right) \\
& \leq N \mu_{1}(t) \sup \left\|z_{s}-z_{s}^{\star}\right\| \\
& \leq N \mu_{1}^{\star}(t)\left\|z-z^{\star}\right\|_{W_{0}}
\end{aligned}
$$

Also, by Definition 2.7 related to phase space and assumption $\left(H_{2}\right)$, let $\mu_{1}^{\star}(t)=\sup _{t \in[0, b]} \mu_{1}(t)$. Then, since $z_{0}=0$ we have:

$$
\begin{aligned}
& \left\|f\left(t, x(t), x_{t}\right)-f\left(t, x^{\star}(t), x_{t}^{\star}\right)\right\|=\left\|f\left(t, y(t)+z(t), y_{t}+z_{t}\right)-f\left(t, y(t)+z^{\star}(t), y_{t}+z_{t}^{\star}\right)\right\| \\
& \leq C_{1}\left\|z(t)-z^{\star}(t)\right\|_{W_{0}}+C_{2}\left\|z_{t}-z_{t}^{\star}\right\|_{\mathcal{P}} \\
& \leq C_{1}\left\|z(t)-z^{*}(t)\right\|_{W_{0}}+C_{2}\left(\mu_{1}(t) \sup _{s \in[0, b]}^{\left\|z_{s}-z_{s}^{*}\right\|}\right. \\
& \left.+\mu_{2}(t)\left\|z_{0}-z_{0}^{\star}\right\|_{\mathcal{P}}\right) \\
& \leq C_{1}\left\|z(t)-z^{\star}(t)\right\|_{W_{0}}+C_{2} \mu_{1}^{\star}(t)\left\|z-z^{*}\right\|_{W_{0}} \\
& \leq\left(C_{1}+C_{2} \mu_{1}^{*}(t)\right)\left\|z-z^{\star}\right\|_{W_{0}}
\end{aligned}
$$


By (54) and (55), we obtain:

$$
\begin{aligned}
\left\|G z(t)-G z^{*}(t)\right\| \leq & \left.N \mu_{1}^{\star}(t)\right)\left\|z-z^{*}\right\|_{W_{0}}+\frac{\alpha\|A\| K N \mu_{1}^{\star}}{\Gamma(\gamma+1)}\left\|z-z^{\star}\right\|_{W_{0}} \\
& \int_{0}^{t}(\psi(t)-\psi(s))^{\alpha-1} \psi^{\prime}(t) d s+\frac{\alpha K\left(C_{1}+C_{2} \mu_{1}^{\star}\right)}{\Gamma(\gamma+1)}\left\|z-z^{*}\right\|_{W_{0}} \\
& \int_{0}^{t}(\psi(t)-\psi(s))^{\alpha-1} \psi(t) d s \\
= & \left.N \mu_{1}^{\star}(t)\right)\left\|z-z^{*}\right\|_{W_{0}}+\frac{\alpha\|A\| K N \mu_{1}^{\star}}{\alpha \Gamma(\gamma+1)} \psi(t)^{\alpha}\left\|z-z^{*}\right\|_{W_{0}} \\
& +\frac{\alpha K\left(C_{1}+C_{2} \mu_{1}^{*}\right)}{\alpha \Gamma(\gamma+1)} \psi(t)^{\alpha}\left\|z-z^{*}\right\|_{W_{0}} \\
\leq & \left(N \mu_{1}^{\star}+\frac{\|A\| K N \mu_{1}^{\star}}{\Gamma(\gamma+1)} \psi(b)^{\alpha}+\frac{K\left(C_{1}+C_{2} \mu_{1}^{*}\right)}{\Gamma(\gamma+1)} \psi(b)^{\alpha}\right)\left\|z-z^{*}\right\|_{W_{0}} \\
\leq & L_{\alpha}\left\|z-z^{*}\right\|_{W_{0}}
\end{aligned}
$$

that is,

$$
\left\|G(z)-G\left(z^{\star}\right)\right\|_{W_{0}} \leq L_{\alpha}\left\|z-z^{\star}\right\|_{W_{0}} .
$$

Hence, taking into account assumptions $\left(H_{1}\right)$ and $\left(H_{2}\right)$, we deduce by the Banach's contraction mapping principle (Lemma 3.1) that $G$ has a unique fixed point $z \in W_{0}$, and obviously, (42) is the mild solution to problem (1) on $(-\infty, b]$.

Now, we assume the following:

$$
\begin{array}{ll}
\left(H_{3}\right): \quad & f:[0, b] \times \mathcal{P} \times \mathbb{X} \longrightarrow \mathbb{X} \text { is a continuous function and there exists } \\
& C_{1}, C_{2}:[0, b] \longrightarrow[0, \infty), \text { such that for any }(t, x, y) \in I \times \mathcal{P} \times \mathbb{X} \\
& \|f(t, x, y)\| \leq C_{1}(t)\|x\|_{\mathcal{P}}+C_{2}(t)\|y\| \\
\left(H_{4}\right): \quad & \text { The function } f:[0, b] \times \mathcal{P} \times \mathbb{X} \longrightarrow \mathbb{X} \text { is a completely continuous. } \\
& h:[0, b] \times \mathcal{P} \longrightarrow \mathbb{X} \text { is a continuous function and there exists } N>0 \\
\left(H_{5}\right): \quad & \text { such that for any }\left(t, x_{t}\right) \in I \times \mathcal{P}
\end{array}
$$

We need to state and prove the following lemmas and the Leray-Schauder alternative theorem in order to prove the next theorem.

Lemma 3.5. Let $\mu_{1}^{\star}=\sup _{s \in[0, b]} \mu_{1}(s), \mu_{2}^{\star}=\sup _{s \in[0, b]} \mu_{2}(s), C_{1}^{\star}=\sup _{s \in[0, b]} C_{1}(s)$ and $C_{2}^{\star}=\sup _{s \in[0, b]} C_{2}(s)$. Then, since $z_{0}=0$, by using the Definition 2.7 related to phase space and assumption $\left(H_{3}\right)-\left(H_{5}\right)$ we have:

$$
\begin{array}{r}
h\left(t, y_{t}+z_{t}\right) \leq N\left(\mu_{2}^{\star}\|\phi\|_{\mathcal{P}}+\mu_{1}^{\star} \sup _{s \in[0, b]} z(s)\right) \\
f\left(t, y(t)+z(t), y_{t}+z_{t}\right) \leq C_{1}^{\star} H+C_{2}^{\star}\left(\mu_{2}^{\star}\|\phi\|_{\mathcal{P}}+\mu_{1}^{\star} \sup _{s \in[0, b]} z(s)\right)
\end{array}
$$


Proof.

$$
\begin{aligned}
\left\|y_{t}+z_{t}\right\|_{\mathcal{P}} & \leq\left\|y_{t}\right\|_{\mathcal{P}}+\left\|z_{t}\right\|_{\mathcal{P}} \\
& \leq \mu_{1}(t) \sup _{s \in[0, b]}\|y(s)\|+\mu_{2}(t)\left\|y_{0}\right\|_{\mathcal{P}}+\mu_{1}(t) \underset{s \in[0, b]}{\sup \|z(s)\|+\mu_{2}(t)\left\|z_{0}\right\|_{\mathcal{P}}} \\
& \leq \mu_{2}(t)\|\phi\|_{\mathcal{P}}+\mu_{1}(t) \sup _{s \in[0, b]}\|z(s)\| \\
& \leq \mu_{2}^{\star}\|\phi\|_{\mathcal{P}}+\mu_{1}^{\star} \sup _{s \in[0, b]}\|z(s)\|
\end{aligned}
$$

On the other hand,

$$
\begin{aligned}
\|y(t)+z(t)\|_{\mathcal{P}} \leq & H\left(\left\|y_{t}\right\|_{\mathcal{P}}+\left\|z_{t}\right\|_{\mathcal{P}}\right) \\
\leq & H\left(\mu_{1}(t) \sup _{s \in[0, b]}\|y(s)\|+\mu_{2}(t)\left\|y_{0}\right\|_{\mathcal{P}}+\mu_{1}(t) \underset{s \in[0, b]}{\sup _{s}\|z(s)\|}\right. \\
& \left.+\mu_{2}(t)\left\|z_{0}\right\|_{\mathcal{P}}\right) \\
\leq & H\left(\mu_{2}(t)\|\phi\|_{\mathcal{P}}+\mu_{1}(t) \sup _{s \in[0, b]}\|z(s)\|\right) \\
\leq & H\left(\mu_{2}^{*}\|\phi\|_{\mathcal{P}}+\mu_{1}^{*} \sup _{s \in[0, b]}\|z(s)\|\right)
\end{aligned}
$$

Therefore, we obtain:

$$
\begin{aligned}
h\left(t, y_{t}+z_{t}\right) & \leq N\left\|y_{t}+z_{t}\right\|_{\mathcal{P}} \\
& \leq N\left(\mu_{2}^{\star}\|\phi\|_{\mathcal{P}}+\mu_{1}^{\star} \sup _{s \in[0, b]}\|z(s)\|\right)
\end{aligned}
$$

and,

$$
\begin{aligned}
f\left(t, y(t)+z(t), y_{t}+z_{t}\right) \leq & C_{1}(t)\|y(t)+z(t)\|_{\mathcal{P}}+C_{2}(t)\left\|y_{t}+z_{t}\right\|_{\mathcal{P}} \\
\leq & C_{1}(t) H\left(\mu_{2}^{\star}\|\phi\|_{\mathcal{P}}+\mu_{1}^{\star} \sup _{s \in[0, b]}\|z(s)\|+C_{2}(t)\right. \\
& \left(\mu_{2}^{\star}\|\phi\|_{\mathcal{P}}+\mu_{1}^{\star} \sup _{s \in[0, b]}\|z(s)\|\right. \\
\leq & C_{1}^{\star} H+C_{2}^{\star}\left(\mu_{2}^{\star}\|\phi\|_{\mathcal{P}}+\mu_{1}^{\star} \sup _{s \in[0, b]}\|z(s)\|\right)
\end{aligned}
$$

Lemma 3.6. (Gronwall type Inequality) Suppose $b \geq 0, \alpha>0$ and $a(t)$ is a nonnegative function locally integrable on $0 \leq t \leq T$ (for some $T \leq+\infty$ ), and suppose $u(t$ ) is nonnegative and locally integrable on $0 \leq t \leq T$ with

$$
u(t) \leq a(t)+b \int_{0}^{t}(t-s)^{\alpha-1} u(s) d s
$$

On this interval, then

$$
u(t) \leq a(t)+\int_{0}^{t}\left[\sum_{n=1}^{\infty} \frac{(b \Gamma(\alpha))^{n}}{\Gamma(n \alpha)}(t-s)^{n \alpha-1} a(s)\right] d s .
$$

Proof. The proof of this lemma can be found in [10, 25].

Theorem 3.2. Let $D \subset H$ be a convex set, $U$ an open subset in $D$ such that $0 \in U$. Then each continuous compact mapping $f: U \longrightarrow D$ has at least one of the following properties: 
(1) $f$ has a fixed-point,

(2) There is $\left(x_{\star}, \lambda_{\star}\right) \in \partial U \times(0,1)$ such that $x_{\star}=\lambda_{\star} f\left(x_{\star}\right)$.

The proof of this lemma can be found in [3,13].

Theorem 3.3. Assume that $\left(H_{3}\right)-\left(H_{5}\right)$ hold and for each bounded set $B$, the set $\left\{t \rightarrow h\left(t, z_{t}\right): z \in B\right\}$ is equicontinuous. Then problem (1) has at least one mild solution on $(-\infty, b]$.

Proof. To prove this result, we aim to use the Leray-Schauder Alternative Theorem based on the following five steps:

Let $G: W_{0} \longrightarrow W_{0}$ be defined as in Theorem 3.1 and let A be a bounded linear operator on $\mathbb{X}$.

Step (1): To show that $G$ is continuous, let $\left(z^{n}\right)$ be a sequence such that $z^{n} \longrightarrow z$ in $W_{0}$ as $n \longrightarrow \infty$. Since $f$ and $h$ are continuous, we have:

$$
\begin{aligned}
f\left(., y(.)+z^{n}(.), y_{(.)}+z_{(.)}^{n}\right) & \longrightarrow f\left(., y(.)+z(.), y_{(.)}+z_{(.)}\right) \\
h\left(., y_{(.)}+z_{(.)}^{n}\right) & \longrightarrow h\left(., y_{(.)}+z_{(.)}\right)
\end{aligned}
$$

Now, for all $t \in[0, b]$ by assumptions and Lemma (3.5), we obtain:

$$
\begin{aligned}
\left\|G\left(z^{n}\right)(t)-G(z)(t)\right\| \leq & \| h\left(t, y_{t}+z_{t}^{n}\right)-h\left(t, y_{t}+z_{t}\right)+\int_{0}^{t}(\psi(t)-\psi(s))^{\alpha-1} \\
& A R_{\psi}^{\alpha}(t-s)\left(h\left(s, y_{s}+z_{s}^{n}\right)-h\left(s, y_{s}+z_{s}\right)\right) \psi^{\prime}(s) d s \\
& +\int_{0}^{t}(\psi(t)-\psi(s))^{\alpha-1} R_{\psi}^{\alpha}(t-s)\left(f \left(s, y(s)+z^{n}(s),\right.\right. \\
& \left.\left.y_{s}+z_{s}^{n}\right)-f\left(s, y(s)+z(s), y_{s}+z_{s}\right)\right) \psi^{\prime}(s) d s \| \\
\leq & \left\|h\left(t, y_{t}+z_{t}^{n}\right)-h\left(t, y_{t}+z_{t}\right)\right\|+\int_{0}^{t}(\psi(t)-\psi(s))^{\alpha-1} \\
& \|A\|\left\|R_{\psi}^{\alpha}(t-s)\right\|\left\|h\left(s, y_{s}+z_{s}^{n}\right)-h\left(s, y_{s}+z_{s}\right)\right\| \psi^{\prime}(s) d s \\
& +\int_{0}^{t}(\psi(t)-\psi(s))^{\alpha-1}\left\|R_{\psi}^{\alpha}(t-s)\right\| \| f\left(s, y(s)+z^{n}(s),\right. \\
& \left.y_{s}+z_{s}^{n}\right)-f\left(s, y(s)+z(s), y_{s}+z_{s}\right) \| \psi^{\prime}(s) d s \\
\leq & N\left(\left\|\left(y_{t}+z_{t}^{n}\right)-\left(y_{t}+z_{t}\right)\right\|_{\mathcal{P}}+\frac{\alpha\|A\| K N}{\Gamma(\alpha+1)}\right. \\
& \int_{0}^{t}(\psi(t)-\psi(s))^{\alpha-1}\left\|\left(y_{s}+z_{s}^{n}\right)-\left(y_{s}+z_{s}\right)\right\|_{\mathcal{P}} \psi^{\prime}(s) d s \\
& +\frac{\alpha\|A\| K}{\Gamma(\alpha+1)} \int_{0}^{t}(\psi(t)-\psi(s))^{\alpha-1}\left[C_{1}(s) \|\left(y(s)+z^{n}(s)\right)\right. \\
& \left.(y(s)+z(s))\left\|_{\mathcal{P}}+C_{2}(s)\right\|\left(y_{s}+z_{s}^{n}\right)-\left(y_{s}+z_{s}\right) \|\right] \psi^{\prime}(s) d s
\end{aligned}
$$




$$
\begin{aligned}
\leq & N\left[2 \mu_{2}^{\star}\|\phi\|_{\mathcal{P}}+\mu_{1}^{\star}\left\|z^{n}-z\right\|_{W_{0}}+2 \mu_{1}^{\star}\|z\|_{W_{0}}\right]+\frac{\alpha\|A\| K}{\Gamma(\alpha+1)} \\
& \int_{0}^{t}(\psi(t)-\psi(s))^{\alpha-1}\left[2 \mu_{2}^{\star}\|\phi\|_{\mathcal{P}}+\mu_{1}^{\star}\left\|z^{n}-z\right\|_{W_{0}}+2 \mu_{1}^{\star}\|z\|_{W_{0}}\right] \psi^{\prime}(s) d s \\
& +\frac{\alpha K}{\Gamma(\alpha+1)}\left[C_{1}^{\star} H+C_{2}^{\star}\left(2 \mu_{2}^{\star}\|\phi\|_{\mathcal{P}}+\mu_{1}^{\star}\left\|z^{n}-z\right\|_{W_{0}}+2 \mu_{1}^{\star}\|z\|_{W_{0}}\right)\right] \\
& \int_{0}^{t}(\psi(t)-\psi(s))^{\alpha-1} \psi^{\prime}(s) d s
\end{aligned}
$$

Since $z^{n} \longrightarrow z$ in $W_{0}$, we know that there exists $\epsilon>0$ such that $\left\|z^{n}-z\right\|_{W_{0}} \leq \epsilon$ for all $n$ to be large enough. Therefore,

$$
\begin{aligned}
\left\|G\left(z^{n}\right)(t)-G(z)(t)\right\|_{W_{0}} \leq & N+\frac{\|A\| K N}{\Gamma(\alpha+1)} \psi(b)^{\alpha}+\frac{K\left(C_{1}^{\star} H+C_{2}^{\star}\right)}{\Gamma(\alpha+1)} \psi(b)^{\alpha} \\
& {\left[2 \mu_{2}^{\star}\|\phi\|_{\mathcal{P}}+\mu_{1}^{\star}\left\|z^{n}-z\right\|_{W_{0}}+2 \mu_{1}^{\star}\|z\|_{W_{0}}\right] } \\
\leq & N+\frac{\|A\| K N}{\Gamma(\alpha+1)} \psi(b)^{\alpha}+\frac{K\left(C_{1}^{\star} H+C_{2}^{\star}\right)}{\Gamma(\alpha+1)} \psi(b)^{\alpha} \\
& {\left[2 \mu_{2}^{\star}\|\phi\|_{\mathcal{P}}+\mu_{1}^{\star} \epsilon+2 \mu_{1}^{\star}\|z\|_{W_{0}}\right] } \\
\leq & \sigma\left[N+\frac{\|A\| K N}{\Gamma(\alpha+1)} \psi(b)^{\alpha}+\frac{K\left(C_{1}^{\star} H+C_{2}^{\star}\right)}{\Gamma(\alpha+1)} \psi(b)^{\alpha}\right]
\end{aligned}
$$

Since $\sigma \in L^{1}\left([0, b], \mathbb{R}_{+}\right)$and based on (64) and (65), $\lim \left\|G\left(z^{n}\right)(t)-G(z)(t)\right\|_{W_{0}} \longrightarrow 0$ as $n \longrightarrow \infty$ which shows that $G$ is continuous.

Step (2): To show that $G$ maps bounded sets of $W_{0}$ into bounded sets in $W_{0}$, for any $q>0$, we set $\mathcal{P}_{q}=$ $\left\{z \in w_{0}\right.$ such that $\left.\|z\|_{W_{0}} \leq q\right\}$. Then, for any $z \in \mathcal{P}$ by assumptions and Lemma (3.5), we have:

$$
\begin{aligned}
\|G(z)(t)\| \leq & \left\|Q_{\psi}^{\alpha}(t) h_{0}\right\|+\left\|h\left(t, y_{t}+z_{t}\right)\right\|+\int_{0}^{t}(\psi(t)-\psi(s))^{\alpha-1} \\
& \|A\|\left\|R_{\psi}^{\alpha}(t-s)\right\|\left\|h\left(s, y_{s}+z_{s}\right)\right\| \psi^{\prime}(s) d s+\int_{0}^{t}(\psi(t) \\
& -\psi(s))^{\alpha-1}\left\|R_{\psi}^{\alpha}(t-s)\right\|\left\|f\left(s, y(s)+z(s), y_{s}+z_{s}\right)\right\| \psi^{\prime}(s) d s \\
\|G(z)(t)\| \leq & K\left\|h_{0}\right\|+N+\frac{\|A\| K N}{\Gamma(\alpha+1)} \psi(b)^{\alpha}+\frac{K\left(C_{1}^{\star} H+C_{2}^{\star}\right)}{\Gamma(\alpha+1)} \psi(b)^{\alpha} \\
& {\left[\mu_{2}^{\star}\|\phi\|_{\mathcal{P}}+\mu_{1}^{\star} \sup _{s \in[0, b]}\|z(s)\|\right] }
\end{aligned}
$$

By using the fact that $z \in \mathcal{P}_{q}$, we obtain:

$$
\mu_{2}^{\star}\|\phi\|_{\mathcal{P}}+\mu_{1}^{\star} \sup _{s \in[0, b]}\|z(s)\| \leq \mu_{2}^{\star}\|\phi\|_{\mathcal{P}}+\mu_{1}^{\star}\|z\|_{W_{0}}=\gamma
$$


Hence,

$$
\|G(z)(t)\|_{W_{0}} \leq K\left\|h_{0}\right\|+\gamma\left[N+\frac{\|A\| K N}{\Gamma(\alpha+1)} \psi(b)^{\alpha}+\frac{K\left(C_{1}^{\star} H+C_{2}^{\star}\right)}{\Gamma(\alpha+1)} \psi(b)^{\alpha}\right]
$$

Now, there exists a positive number $\lambda$ such that

$$
\lambda=K\left\|h_{0}\right\|+\gamma\left[N+\frac{\|A\| K N}{\Gamma(\alpha+1)} \psi(b)^{\alpha}+\frac{K\left(C_{1}^{\star} H+C_{2}^{\star}\right)}{\Gamma(\alpha+1)} \psi(b)^{\alpha}\right]
$$

Thus, $\|G(z)(t)\|_{W_{0}} \leq \lambda$. This proves that $G\left(\mathcal{P}_{q}\right) \subset \mathcal{P}_{\lambda}$

Step (3): To show that $G$ maps bounded sets of $W_{0}$ into equi-continuous sets of $W_{0}$, let $q$ be defined as in Step(2). Let $t_{1}, t_{2} \in[0, b], t_{1}>t_{2}$ and $z \in \mathcal{P}_{q}$ and assume that for each bounded set $B$, the set $\left\{t \rightarrow h\left(t, z_{t}\right)\right.$ : $z \in B\}$ is equicontinuous. Then,

$$
\begin{aligned}
\left\|G(z)\left(t_{1}\right)-G(z)\left(t_{2}\right)\right\| \leq & \left\|Q_{\psi}^{\alpha}\left(t_{1}-t_{2}\right) h_{0}\right\|+\left\|h\left(t_{1}, y_{t_{1}}+z_{t_{1}}\right)-h\left(t_{2}, y_{t_{2}}+z_{t_{2}}\right)\right\| \\
& +\int_{0}^{t_{2}}\left(\left(\psi\left(t_{1}\right)-\psi(s)\right)^{\alpha-1}-\left(\psi\left(t_{2}\right)-\psi(s)\right)^{\alpha-1}\right)\|A\| \\
& \left\|R_{\psi}^{\alpha}\left(t_{1}-s\right)-R_{\psi}^{\alpha}\left(t_{2}-s\right)\right\|\left\|h\left(s, y_{s}+z_{s}\right)\right\| \psi(s) d s \\
& +\int_{t_{2}}^{t_{1}}\left(\psi\left(t_{1}\right)-\psi(s)\right)^{\alpha-1}\|A\|\left\|R_{\psi}^{\alpha}\left(t_{1}-s\right)\right\| \\
& \left\|h\left(s, y_{s}+z_{s}\right)\right\| \psi^{\prime}(s) d s+\int_{0}^{t_{2}}\left(\left(\psi\left(t_{1}\right)-\psi(s)\right)^{\alpha-1}\right. \\
& \left.-\left(\psi\left(t_{2}\right)-\psi(s)\right)^{\alpha-1}\right)\|A\|\left\|R_{\psi}^{\alpha}\left(t_{1}-s\right)-R_{\psi}^{\alpha}\left(t_{2}-s\right)\right\| \\
& \left\|f\left(s, y(s)+z(s), y_{s}+z_{s}\right)\right\| \psi(s) d s+\int_{t_{2}}^{t_{1}}\left(\psi\left(t_{1}\right)-\psi(s)\right)^{\alpha-1} \\
& \|A\|\left\|R_{\psi}^{\alpha}\left(t_{1}-s\right)\right\|\left\|f\left(s, y(s)+z(s), y_{s}+z_{s}\right)\right\| \psi(s) d s
\end{aligned}
$$


Since $t_{1} \longrightarrow t_{2}$ independently of $z \in \mathcal{P}_{q}$, by assumptions and Lemma (3.5), we obtain:

$$
\begin{aligned}
& \left\|G(z)\left(t_{1}\right)-G(z)\left(t_{2}\right)\right\| \leq\left\|Q_{\psi}^{\alpha}\left(t_{1}-t_{2}\right) h_{0}\right\|+N\left(\mu_{2}^{\star}\|\phi\|_{\mathcal{P}}+\mu_{1}^{\star} \sup \left\|z\left(s_{1}\right)-z\left(s_{2}\right)\right\|\right) \\
& +\frac{\alpha\|A\| K N}{\Gamma(\alpha+1)}\left[\int_{0}^{t_{2}}\left(\left(\psi\left(t_{1}\right)-\psi(s)\right)^{\alpha-1}-\left(\psi\left(t_{2}\right)-\psi(s)\right)^{\alpha-1}\right)\right. \\
& \left(\mu_{2}^{\star}\|\phi\|_{\mathcal{P}}+\mu_{1}^{\star} \sup _{\tau \in[0, b]}\|z(\tau)\|\right) \psi^{\prime}(s) d s \\
& +\int_{t_{2}}^{t_{1}}\left(\psi\left(t_{1}\right)-\psi(s)\right)^{\alpha-1}\left(\mu_{2}^{\star}\|\phi\|_{\mathcal{P}}+\mu_{1}^{\star} \sup _{\tau \in[0, b]}\|z(\tau)\|\right) \\
& \left.\psi^{\prime}(s) d s\right]+\frac{\alpha K}{\Gamma(\alpha+1)}\left[\int _ { 0 } ^ { t _ { 2 } } \left(\left(\psi\left(t_{1}\right)-\psi(s)\right)^{\alpha-1}\right.\right. \\
& \left.-\left(\psi\left(t_{2}\right)-\psi(s)\right)^{\alpha-1}\right)\left(C_{1}^{\star} H+C_{2}^{\star}\left(\mu_{2}^{\star}\|\phi\|_{\mathcal{P}}\right.\right. \\
& \left.\left.+\mu_{1}^{\star} \sup _{\tau \in[0, b]}\|z(\tau)\|\right)\right)+\int_{t_{2}}^{t_{1}}\left(\psi\left(t_{1}\right)-\psi(s)\right)^{\alpha-1} \\
& \left.\left(C_{1}^{\star} H+C_{2}^{\star}\left(\mu_{2}^{\star}\|\phi\|_{\mathcal{P}}+\mu_{1}^{\star} \sup _{\tau \in[0, b]}\|z(\tau)\|\right)\right) \psi^{\prime}(s) d s\right]
\end{aligned}
$$

By using the fact that $z \in \mathcal{P}_{q}$, we obtain:

$$
\mu_{2}^{\star}\|\phi\|_{\mathcal{P}}+\mu_{1}^{\star} \sup _{s \in[0, b]}\|z(s)\| \leq \mu_{2}^{\star}\|\phi\|_{\mathcal{P}}+\mu_{1}^{\star}\|z\|_{W_{0}}=\gamma
$$

Hence,

$$
\begin{aligned}
\left\|G(z)\left(t_{1}\right)-G(z)\left(t_{2}\right)\right\|_{W_{0}} \leq & K\left\|h_{0}\right\|+\gamma\left(N+\frac{\alpha\|A\| K N}{\Gamma(\alpha+1)}\right. \\
& {\left[\int_{0}^{t_{2}}\left(\left(\psi\left(t_{1}\right)-\psi(s)\right)^{\alpha-1}-\left(\psi\left(t_{2}\right)-\psi(s)\right)^{\alpha-1}\right) \psi^{\prime}(s) d s\right.} \\
& \left.+\int_{t_{2}}^{t_{1}}\left(\psi\left(t_{1}\right)-\psi(s)\right)^{\alpha-1} \psi^{\prime}(s) d s\right] \\
& +\frac{\alpha K}{\Gamma(\alpha+1)}\left(C_{1}^{\star} H+C_{2}^{\star}\right)\left[\int _ { 0 } ^ { t _ { 2 } } \left(\left(\psi\left(t_{1}\right)-\psi(s)\right)^{\alpha-1}\right.\right. \\
& \left.-\left(\psi\left(t_{2}\right)-\psi(s)\right)^{\alpha-1}\right) \psi^{\prime}(s) d s \\
& \left.\left.+\int_{t_{2}}^{t_{1}}\left(\psi\left(t_{1}\right)-\psi(s)\right)^{\alpha-1} \psi^{\prime}(s) d s\right]\right)
\end{aligned}
$$

We know from Lemma (3.4) that:

$$
\left\|Q_{\psi}^{\alpha}\left(t_{1}-t_{2}\right)\right\|_{B(x)} \leq K \in L^{1}\left(I, \mathbb{R}_{+}\right)
$$

and,

$$
\left\|R_{\psi}^{\alpha}\left(t_{1}-s\right)-R_{\psi}^{\alpha}\left(t_{2}-s\right)\right\|_{B(x)} \leq \frac{K}{\Gamma(\alpha+1} \in L^{1}\left(I, \mathbb{R}_{+}\right)
$$

Thus, $Q_{\psi}^{\alpha}\left(t_{1}-t_{2}\right) \longrightarrow 0$ and $R_{\psi}^{\alpha}\left(t_{1}-s\right)-R_{\psi}^{\alpha}\left(t_{2}-s\right) \longrightarrow 0$ as $t_{1} \longrightarrow t_{2}$ since $Q_{\psi}^{\alpha}$ and $R_{\psi}^{\alpha}$ are strongly continuous. 
Also, we obtain that:

$\int_{0}^{t_{2}}\left(\left(\psi\left(t_{1}\right)-\psi(s)\right)^{\alpha-1}-\left(\psi\left(t_{2}\right)-\psi(s)\right)^{\alpha-1}\right) \psi^{\prime}(s) d s \longrightarrow 0$

and,

$\int_{t_{2}}^{t_{1}}\left(\psi\left(t_{1}\right)-\psi(s)\right)^{\alpha-1} \psi^{\prime}(s) d s \longrightarrow 0$.

Therefore, $\lim \left\|G(z)\left(t_{1}\right)-G(z)\left(t_{2}\right)\right\|_{W_{0}} \longrightarrow 0$ as $t_{1} \longrightarrow t_{2}$. This proves that $G$ is equi-continuous.

Step (4): we want to show that the operator $G$ maps $\mathcal{P}_{q}$ into a relatively compact set in $\mathbb{X}$. Since $Q_{\psi}^{\alpha}$ and $R_{\psi}^{\alpha}$ are strongly continuous, based on the assumptions, the sets $\left\{Q_{\psi}^{\alpha}(t) h_{0}\right\},\left\{R_{\psi}^{\alpha}(t-s) h\left(s, y_{s}+z_{s}\right)\right\}$ and $\left\{R_{\psi}^{\alpha}(t-s)\right.$ $\left.f\left(s, y(s)+z(s), y_{s}+z_{s}\right)\right\}$ are relatively compact in $\mathbb{X}$. Moreover, by using the mean value theorem for the Bochner integral, for $z \in \mathcal{P}_{q}$ and for $t \in[0, b]$ we have:

$$
\begin{aligned}
G(z)(t) \in t \overline{c o n v} \quad & \left\{Q_{\psi}^{\alpha}(t) h_{0}+h\left(t, y_{t}+z_{t}\right)+\int_{0}^{t}(\psi(t)-\psi(s))^{\alpha-1}\right. \\
& A R_{\psi}^{\alpha}(t-s) h\left(s, y_{s}+z_{s}\right) \psi^{\prime}(s) d s+\int_{0}^{t}(\psi(t)-\psi(s))^{\alpha-1} \\
& \left.R_{\psi}^{\alpha}(t-s) f\left(s, y(s)+z(s), y_{s}+z_{s}\right) \psi^{\prime}(s) d s ; s \in[0, b], z \in \mathcal{P}_{q}\right\}
\end{aligned}
$$

Thus the set $\left\{G(z)(t): z \in \mathcal{P}_{q}\right\}$ is relatively compact in $\mathbb{X}$ for $t \in[0, b]$.

Step (5): Let us set $D=\left\{z \in W_{0}\right.$ such that $\left.z=\lambda G(z)\right\}$ for some $0<\lambda<1$. Let $z \in D$, therefore we have:

$$
\begin{aligned}
(z)(t) \leq & \lambda\left[\left\|Q_{\psi}^{\alpha}(t) h_{0}\right\|+\left\|h\left(t, y_{t}+z_{t}\right)\right\|+\int_{0}^{t}(\psi(t)-\psi(s))^{\alpha-1}\right. \\
& \|A\|\left\|R_{\psi}^{\alpha}(t-s)\right\|\left\|h\left(s, y_{s}+z_{s}\right)\right\| \psi^{\prime}(s) d s+\int_{0}^{t}(\psi(t)-\psi(s))^{\alpha-1} \\
& \left.\left\|R_{\psi}^{\alpha}(t-s)\right\|\left\|f\left(s, y(s)+z(s), y_{s}+z_{s}\right)\right\| \psi^{\prime}(s) d s\right] \\
\leq & K\left\|h_{0}\right\|+\left[N+\frac{\alpha\|A\| K N}{\Gamma(\alpha+1)} \int_{0}^{t}(\psi(t)-\psi(s))^{\alpha-1} \psi^{\prime}(s) d s\right. \\
& \left.+\frac{\alpha K}{\Gamma(\alpha+1)} \int_{0}^{t}(\psi(t)-\psi(s))^{\alpha-1} \psi^{\prime}(s) d s\right] \mu_{2}^{\star}\|\phi\|_{\mathcal{P}} \\
& +\mu_{1}^{\star} \sup _{\tau \in[0, b]}\|z(\tau)\|
\end{aligned}
$$

Let $\tau^{\star} \in[0, s]$ such that for $s \in[0, b]$,

$$
(\psi(t)-\psi(s))^{\alpha-1} \sup _{\tau \in[0, b]}\|z(\tau)\|=(\psi(t)-\psi(s))^{\alpha-1}\left\|z\left(\tau^{\star}\right)\right\|
$$


If $\tau^{\star} \in[0, b]$, we have:

$$
\begin{aligned}
\|(z)(t)\| \leq & K\left\|h_{0}\right\|+\left[N+\frac{\alpha\|A\| K N}{\Gamma(\alpha+1)} \int_{0}^{t}(\psi(t)-\psi(s))^{\alpha-1} \psi^{\prime}(s) d s\right. \\
& \left.+\frac{\alpha K}{\Gamma(\alpha+1)} \int_{0}^{t}(\psi(t)-\psi(s))^{\alpha-1} \psi^{\prime}(s) d s\right]\left(\mu_{2}^{\star}\|\phi\|_{\mathcal{P}}\right) \\
& +N \mu_{1}^{\star}\|z(s)\|+\left(\frac{\alpha\|A\| K N}{\Gamma(\alpha+1)}+\frac{\alpha K}{\Gamma(\alpha+1)}\right) \\
& \int_{0}^{t}(\psi(t)-\psi(s))^{\alpha-1}\|z(s)\| \psi^{\prime}(s) d s
\end{aligned}
$$

by using Lemma (3.6):

$$
\begin{aligned}
\|(z)(t)\|_{W_{0}} \leq & K\left\|h_{0}\right\|+\mu_{2}^{*}\|\phi\|_{\mathcal{P}}\left[N+\frac{\|A\| K N}{\Gamma(\alpha+1)} \psi(b)^{\alpha}\right. \\
& \left.+\frac{K}{\Gamma(\alpha+1)} \psi(b)^{\alpha}\right]+N \mu_{1}^{*}\|z\|_{W_{0}}[1+ \\
& \left.\int_{0}^{t}\left[\sum_{n=1}^{\infty} \frac{\left(\frac{\alpha\|A\| K N}{\Gamma(\alpha+1)}+\frac{\alpha K}{\Gamma(\alpha+1)} \Gamma(\alpha)\right)^{n}}{\Gamma(n \alpha)}((\psi(t)-\psi(s)))^{n \alpha-1}\right] d s\right] \\
\leq & \theta_{1}\left[1+\sum_{n=1}^{\infty} \frac{\left.\theta_{2} \Gamma(\alpha)\right)^{n}}{n \alpha \Gamma(n \alpha)} \psi(b)^{n \alpha}\right] \\
\leq & \theta_{1} E_{\alpha}\left(\theta_{2} \Gamma(\alpha) \psi(b)^{\alpha}\right)
\end{aligned}
$$

where,

$$
\begin{aligned}
\theta_{1} & =K\left\|h_{0}\right\|+\mu_{2}^{\star}\|\phi\|_{\mathcal{P}}\left[N+\frac{\|A\| K N}{\Gamma(\alpha+1)} \psi(b)^{\alpha}+\frac{K}{\Gamma(\alpha+1)} \psi(b)^{\alpha}\right]+N \mu_{1}^{\star}\|z\|_{W_{0}} \\
\theta_{2} & =\frac{\alpha\|A\| K N}{\Gamma(\alpha+1)}+\frac{\alpha K}{\Gamma(\alpha+1)}
\end{aligned}
$$

and,

$$
E_{\alpha}\left(\theta_{2} \Gamma(\alpha) \psi(b)^{\alpha}\right)=\sum_{n=0}^{\infty} \frac{\left.\theta_{2} \Gamma(\alpha) \psi(b)^{\alpha}\right)^{n}}{\Gamma(n \alpha+1)}
$$

which is the Mittage-Leffer function. Thus, we obtain:

$$
\|(z)(t)\|_{W_{0}} \leq \theta_{1} E_{\alpha}\left(\theta_{2} \Gamma(\alpha) \psi(b)^{\alpha}\right)
$$

which proves that $\|(z)(t)\|_{W_{0}}$ is bounded. Therefore, by proving the steps (1)- (5) and using Leray-Schauder Alternative Theorem, we result that $G$ has a fixed point which is a mild solution of problem $(1)$ on $(-\infty, b]$.

Acknowledgments: The authors are grateful to the anonymous reviewers, for their valuable suggestions and careful reading which helped improve this work. 
Conflict of interest: On behalf of all authors, the corresponding author states that there is no conflict of interest.

Data Availability Statement: Data sharing is not applicable to this article as no datasets were generated or analysed during the current study.

\section{References}

[1] M. S. Abdo, S. K. Panchal, S. P. Bhairat, On existence of solution to nonlinear $\psi$-Hilfer Cauchy-type problem, math.GM, arXiv:1909.13681 (2019).

[2] N.T. Anh and T.D. Ke, Decay integral solutions for neutral fractional differential equations with infinite delays, Math. Methods Appl. Sci. 38 (2015), 1601-1622.

[3] J. Dugundji and A. Granas, Fixed Point Theory, Warsaw (1982).

[4] K. J. Engel and R. Nagel, One-parameter semigroups for linear evolution equations, Springer Science \& Business Media, 194 (1999).

[5] H. M. Fahad, M. U. Rehman, A. Fernandez, On Laplace transforms with respect to functions and their applications to fractional differential equations, arXiv:1907.04541 [math.CA] (2020).

[6] R. G. Foko Tiomela, F. Norouzi, G. M. N'Guérékata, G. M. Mophou On the stability and stabilization of some semilinear fractional differential equations in Banach Spaces, Fractional Differential Calculus.10 (2), (2020).

[7] X. Gourdon, Analyse: Mathématiques pour MP*, Ellipses Marketing (2008).

[8] A. Granas and J. Dugundji, Fixed point theory, Science \& Business Media, Springer (2013).

[9] J. Hale and J. Kato, Phase space for retarded equations with infinite delay, Funkcial. Ekvac. 21 (1978), 11-41.

[10] D. Henry, Geometric Theory of Semilinear Parabolic Equations. Lecture Notes in Mathematics, Springer-Verlag, New York/Berlin. 8 (1981).

[11] F. Jarad and T. Abdeljawad, Generalized fractional derivatives and Laplace transform, Discrete \& Continuous Dynamical Systems (2019), 709-722.

[12] A. A. Kilbas, H. M. Srivastava, J. J. Trujillo, Theory and applications of fractional differential equations, elsevier, 204 (2006).

[13] J. Leray and J. Schauder, Topologie et equations fonctionnelles, Ann. Sci. Ecole Norm. Sup. 51 (1934), 45-78.

[14] F. Li, An existence result for fractional differential equations of neutral type with infinite delay, Electron. J. Qual. Theory Differ. Equ., 52 (2011), 1-15.

[15] Liu K. Wang J., O’Regan D, Ulam-Hyers-Mittag-Leffer stability for $\psi$-Hilfer fractional-order delay differential equations. Advances in Difference Equations.50 (2019).

[16] D. Luo, K. Shah, Z. Luo, On the Novel Ulam-Hyers Stability for a Class of Nonlinear $\psi$-Hilfer Fractional Differential Equation with Time-Varying Delays, Mediterr. J. Math. (2019), 16-112.

[17] A. M. Mathai and H.J. Haubold, An Introduction to Fractional Calculus, Mathematics Research Developments, Nova Science Publishers (2017).

[18] G. M. Mophou and G. M. N'Guérékata, Existence of mild solutions of some semilinear neutral fractional functional evolution equations with infinite delay, Appl. Math. Comput., 216 (2010), 61-69.

[19] G.M. Mophou, G. M. N'Guérékata, A note on a semilinear fractional differential equation of neutral type with infinite delay, Adv. Difference Equ. (2010).

[20] F. Norouzi and G.M. N'Guérékata, A new study of fractional-order financial system via homotopy analysis, Analele Universitatii Oradea. Fasc. Matematica. 27 (1), 141-152 (2020).

[21] M. El-Shahed and A. Salem An extension of wright function and its properties, Journal of Mathematics, Hindawi, 2015 (2015).

[22] A. Suechoei, P. S. Ngiamsunthorn, Existence uniqueness and stability of mild solutions for semilinear $\psi$-Caputo fractional evolution equations, Advances in Difference Equations, 114 (2020).

[23] J. Vanterler da C. Sousa and E. Capelas de Oliveira, On the $\psi$-Hilfer fractional derivative. Common Nonlinear Sci Numer Simulat., 60 (2018), 72-91.

[24] B. M. Yambiyo , F. Norouzi and G. M. N'Guerekata. A study of an epidemic SIR model via Homotopy Analysis Method in the sense of Caputo-fractional system. Studies in evolution equations and related topics STEAM-H series, Springer (2021) (to appear).

[25] H. Ye, J. Gao, Y. Ding, A generalized Gronwall inequality and its application to a fractional differential equation. J. Math. Anal. Appl., 338 (2007) 1075-1081.

[26] Y. Zhou, F. Jiao, Nonlocal Cauchy problem for fractional evolution equations, Nonlinear Analysis: Real World Applications, 11 (2010), 4465-4475.

[27] Y. Zhou, F. Jiao, Existence of mild solutions for fractional neutral evolution equations, Computers and Mathematics with Applications, 59 (2010), 1063-1077. 